MATHEMATICS OF COMPUTATION

Volume 67, Number 223, July 1998, Pages 917-946

S $0025-5718(98) 00941-7$

\title{
NONCONFORMING FINITE ELEMENT APPROXIMATION OF CRYSTALLINE MICROSTRUCTURE
}

\author{
BO LI AND MITCHELL LUSKIN
}

\begin{abstract}
We consider a class of nonconforming finite element approximations of a simply laminated microstructure which minimizes the nonconvex variational problem for the deformation of martensitic crystals which can undergo either an orthorhombic to monoclinic (double well) or a cubic to tetragonal (triple well) transformation. We first establish a series of error bounds in terms of elastic energies for the $L^{2}$ approximation of derivatives of the deformation in the direction tangential to parallel layers of the laminate, for the $L^{2}$ approximation of the deformation, for the weak approximation of the deformation gradient, for the approximation of volume fractions of deformation gradients, and for the approximation of nonlinear integrals of the deformation gradient. We then use these bounds to give corresponding convergence rates for quasi-optimal finite element approximations.
\end{abstract}

\section{INTRODUCTION}

The nonconvex elastic energy used to model martensitic crystals is generally minimized only by a microstructure [3], [4], [9], [19], [23], [26], [31]. A common example of such a microstructure is a simple laminate in which the deformation gradient oscillates on a fine or infinitesimal scale in parallel layers between two stress-free homogeneous states.

Finite element approximations of energy-minimizing laminates necessarily have a finite thickness. Although conforming finite element methods can be proven to give convergent approximations to the microstructure [28], [29], [31], [32], they cannot generally give a laminate which oscillates on the scale of the mesh size for arbitrarily oriented meshes [11], [31].

Nonconforming finite element approximations are not required to be globally continuous [10], [38], so it is reasonable to think that they would be able to give a more accurate approximation to fine-scale microstructure [31]. The class of nonconforming finite element methods analyzed in this paper was successfully used to compute crystalline microstructure in [25]. These elements were first proposed, tested, and analyzed in [39] for the Stokes problem. A short discussion on one of these elements in the setting of the mixed finite element method can be found in [2]. This class of elements was analyzed for general second-order elliptic problems in

Received by the editor June 12, 1996 and, in revised form, February 26, 1997.

1991 Mathematics Subject Classification. Primary 49M15, 65C20, 65N30, 73C50, 73K20.

Key words and phrases. Finite element, nonconforming, microstructure, martensitic transformation, error estimate.

This work was supported in part by the NSF through grant DMS 95-05077, by the AFOSR through grants AF/F49620-96-1-0057 and AF/F49620-97-1-0187, by the Institute for Mathematics and its Applications, and by a grant from the Minnesota Supercomputer Institute. 
[24]. In this paper, we prove the convergence of these nonconforming methods to an energy-minimizing microstructure for the nonconvex elastic energies which model martensitic crystals which can undergo either an orthorhombic to monoclinic (double well) transformation or a cubic to tetragonal (triple well) transformation. The results in this paper also hold for a general rotationally invariant, double well energy density.

In the recently developed geometrically nonlinear theory of martensitic crystals, the elastic energy density attains its minimum value (below the transformation temperature) on a set

$$
\mathrm{SO}(3) U_{1} \cup \cdots \cup \mathrm{SO}(3) U_{N},
$$

where $\mathrm{SO}(3)$ is the group of proper rotations defined by

$$
\operatorname{SO}(3)=\left\{Q \in \mathbb{R}^{3 \times 3}: Q^{T}=Q^{-1} \text { and } \operatorname{det} Q=1\right\},
$$

and where the symmetry-related matrices, $U_{1}, \cdots, U_{N}$, for $N>1$, represent the martensitic variants. The martensitic variants $U_{1}, \cdots, U_{N}$ are linear transformations which transform the lattice of the austenitic phase into the lattice of the martensitic phase. In the above, $\mathbb{R}^{3 \times 3}$ is the set of all $3 \times 3$ real matrices.

A martensitic crystal which can undergo an orthorhombic to monoclinic transformation has two symmetry-related martensitic variants, that is, $N=2$, and hence the elastic energy density has two wells [4], [31]. A more commonly observed martensitic transformation is the cubic to tetragonal transformation [3], [4], [31]. In this case, there are three associated symmetry-related martensitic variants, so $N=3$, and the elastic energy density has therefore three wells.

For certain boundary conditions, the elastic energy of the martensitic crystal cannot be minimized by a deformation and can be lowered as much as possible only by a sequence of deformations whose gradients oscillate so that the limiting volume fraction is nonzero for more than one gradient [4], [31]. Based on the hypothesis that the crystal structure is determined by the principle of energy minimization, the geometrically nonlinear theory describes the crystalline microstructure as the limiting configuration of energy-minimizing sequences of deformations [3], [4], [9], [19], [23], [26], [31].

Both of our nonconforming finite elements are defined on rectangular parallelepipeds. The first one has its degrees of freedom given by the values at the centers of the faces of the rectangular parallelepipeds. The second one has its degrees of freedom given by the averages over the six faces of the rectangular parallelepipeds. To prove the convergence of this class of nonconforming finite element methods for the nonconvex energies which model crystalline microstructure, we prove some important properties of the nonconforming finite element deformations. They include a discrete version of a slight variation of the divergence theorem, a Poincare type inequality which is more general than that in [24], and a discrete version of the usual trace theorem in Sobolev spaces [1]. These properties will be used as key technical tools in establishing various kinds of error bounds in terms of the elastic energy.

Our analysis utilizes the theory of numerical analysis for the microstructure in nonconvex variational problems that was developed in [13], [16], and extended in $[6],[7],[8],[21],[34]$. This theory was also used to analyze the finite element approximation of microstructure in micromagnetics [33]. The approximation of relaxed variational problems has been analyzed in [5], [20], [35], [36], [37], [40], [41]. 
A nonconforming finite element approximation for a nonconvex variational problem with not only an elastic energy but also a nonphysical penalty term was analyzed in $[21]$.

An analysis of the finite element approximation for a physical, rotationally invariant energy was first given in [32] for the orthorhombic to monoclinic transformation. This analysis has been extended to the cubic to orthorhombic transformation [29], to more general boundary conditions [27], [28], and to the method of reduced integration [12]. The estimates in these papers and in this paper show that all of the local minima of the energy (restricted to the finite element space) which satisfy a quasi-optimality condition give accurate approximations to the energy-minimizing microstructure for the deformation, the volume fractions of the deformation gradients, and the nonlinear integrals of the deformation gradient.

In this paper, we further generalize the results in [29], [32] to the approximation by the two nonconforming finite elements. Our results show that the approximation errors due to the nonconformity of the employed nonconforming finite elements are negligible compared with the errors of the approximation of microstructure which are already present in the conforming approximation. Therefore, the asymptotic rate of convergence that we obtain for the nonconforming methods is equal to the rate found for the conforming methods.

We refer to [31] for an introduction to the modeling and computation of crystalline microstructure and for a more extensive survey of results and references.

We organize the rest of the paper as follows. In $\S 2$, we describe the underlying continuum model for crystals which can undergo either an orthorhombic to monoclinic or a cubic to tetragonal martensitic transformation. In $\S 3$, we review the definition and basic properties of the class of nonconforming finite element spaces that we analyze. Further properties of nonconforming finite element deformations are given in $\S 4$. These properties are then used to establish a series of error bounds in terms of the elastic energy for the nonconforming finite element approximations in $\S 5-\S 7$. Finally, in $\S 8$, we first prove the existence of finite element energy minimizers and then derive the corresponding error estimates for quasi-optimal nonconforming finite element approximations.

\section{Multi-Well EnERGy minimization PROBlems}

We first briefly review some basic definitions and properties of martensitic crystals which can undergo either an orthorhombic to monoclinic or a cubic to tetragonal phase transformation. For more details, we refer to [3], [4], [31].

The energy wells for an orthorhombic to monoclinic transformation are determined by the martensitic variants

$$
U_{1}=\left(I+\eta e_{2} \otimes e_{1}\right) D, \quad U_{2}=\left(I-\eta e_{2} \otimes e_{1}\right) D,
$$

where $I$ is the identity transformation from $\mathbb{R}^{3}$ to $\mathbb{R}^{3}, \eta>0$ is a material parameter, $\left\{e_{1}, e_{2}, e_{3}\right\}$ is an orthonormal basis for $\mathbb{R}^{3}$, and $D$ is a diagonal, positive definite, linear transformation given by

$$
D=d_{1} e_{1} \otimes e_{1}+d_{2} e_{2} \otimes e_{2}+d_{3} e_{3} \otimes e_{3}
$$

for some $d_{1}, d_{2}, d_{3}>0$. We recall that the tensor $a \otimes n$ for $a, n \in \mathbb{R}^{3}$ defines the linear transformation $(a \otimes n) v=(n \cdot v) a$ for $v \in \mathbb{R}^{3}$. 
The energy wells for a cubic to tetragonal transformation are determined by the martensitic variants

$$
\begin{gathered}
U_{1}=\eta_{1} I+\left(\eta_{2}-\eta_{1}\right) e_{1} \otimes e_{1}, \quad U_{2}=\eta_{1} I+\left(\eta_{2}-\eta_{1}\right) e_{2} \otimes e_{2}, \\
U_{3}=\eta_{1} I+\left(\eta_{2}-\eta_{1}\right) e_{3} \otimes e_{3},
\end{gathered}
$$

where $\eta_{1}>0$ and $\eta_{2}>0$ are material parameters such that $\eta_{1} \neq \eta_{2}$, and $\left\{e_{1}, e_{2}, e_{3}\right\}$ is again an orthonormal basis for $\mathbb{R}^{3}$.

For convenience, we define the set of indices $K$ to be $K=\{1,2\}$ for the orthorhombic to monoclinic transformation and $K=\{1,2,3\}$ for the cubic to tetragonal transformation. We also denote

$$
\mathcal{U}_{i}=\mathrm{SO}(3) U_{i}, \quad i \in K, \quad \text { and } \quad \mathcal{U}=\bigcup\left\{\mathcal{U}_{i}: i \in K\right\} .
$$

The following lemma, proved in [3], [4], [31], serves as a key crystallographical basis for our analysis.

Lemma 2.1. (1) For each $i \in K$ there is no rank-one connection between $\mathcal{U}_{i}$ and itself, that is, there do not exist $F_{0}, F_{1} \in \mathcal{U}_{i}$ with $F_{0} \neq F_{1}$ such that

$$
F_{1}=F_{0}+a \otimes n
$$

for some $a \in \mathbb{R}^{3}$ and $n \in \mathbb{R}^{3},|n|=1$.

(2) For any $i, j \in K, i \neq j$, there are exactly two rank-one connections between $\mathcal{U}_{i}$ and $\mathcal{U}_{j}$, that is, for any $F_{0} \in \mathcal{U}_{i}$ there are exactly two distinct $F_{1} \in \mathcal{U}_{j}$ such that

$$
F_{1}=F_{0}+a \otimes n
$$

for some $a \in \mathbb{R}^{3}$ and $n \in \mathbb{R}^{3},|n|=1$. In this case, we have also for any $\lambda \in(0,1)$ that

$$
(1-\lambda) F_{0}+\lambda F_{1} \notin \mathcal{U}
$$

Moreover, we have for the orthorhombic to monoclinic transformation that

$$
n \in\left\{ \pm e_{1}, \pm e_{2}\right\},
$$

and for the cubic to tetragonal transformation that

$$
n \in\left\{ \pm \frac{1}{\sqrt{2}}\left(e_{i}+e_{j}\right), \pm \frac{1}{\sqrt{2}}\left(e_{i}-e_{j}\right)\right\} .
$$

We now consider a crystal that can undergo either an orthorhombic to monoclinic or a cubic to tetragonal transformation. We denote by $\Omega$ the reference configuration of the crystal which is taken to be the homogeneous austenitic phase at the transformation temperature. We assume that $\Omega \subset \mathbb{R}^{3}$ is a bounded domain with a Lipschitz continuous boundary. We denote deformations by $y: \Omega \rightarrow \mathbb{R}^{3}$ and corresponding deformation gradients by $\nabla y: \Omega \rightarrow \mathbb{R}^{3 \times 3}$. We denote the elastic energy density at a fixed temperature below the transformation temperature by the continuous function $\phi: \mathbb{R}^{3 \times 3} \rightarrow \mathbb{R}$. The elastic energy of a deformation $y$ is then given by

$$
\mathcal{E}(y) \equiv \int_{\Omega} \phi(\nabla y(x)) d x
$$

To model the underlying martensitic transformations, we assume that the energy density $\phi$ is minimized on the energy wells $\mathcal{U}_{i}=\mathrm{SO}(3) U_{i}, i \in K$, so we assume (after 
adding a constant to the energy density) that

$$
\begin{gathered}
\phi(F) \geq 0, \quad \forall F \in \mathbb{R}^{3 \times 3}, \\
\phi(F)=0 \quad \text { if and only if } \quad F \in \mathcal{U}=\bigcup\left\{\mathcal{U}_{i}: i \in K\right\} .
\end{gathered}
$$

We shall also assume that the energy density $\phi$ grows quadratically away from the energy wells, that is,

$$
\phi(F) \geq \kappa\|F-\pi(F)\|^{2}, \quad \forall F \in \mathbb{R}^{3 \times 3},
$$

where $\kappa>0$ is a constant and $\pi: \mathbb{R}^{3 \times 3} \rightarrow \mathcal{U}$ is a Borel measurable projection defined by

$$
\|F-\pi(F)\|=\min _{G \in \mathcal{U}}\|F-G\|, \quad \forall F \in \mathbb{R}^{3 \times 3} .
$$

In the above and in the following we use the matrix norm defined by

$$
\|F\|^{2}=\operatorname{trace}\left(F^{T} F\right)=\sum_{i, j=1}^{3} F_{i j}^{2}, \quad \forall F=\left(F_{i j}\right) \in \mathbb{R}^{3 \times 3} .
$$

The projection $\pi(F)$ exists for any $F \in \mathbb{R}^{3 \times 3}$ since $\mathcal{U}$ is compact, although the projection may not be unique. It is unique, however, if $\|F-\pi(F)\|$ is small enough [31].

Let $F_{0}, F_{1} \in \mathcal{U}$ be rank-one connected so as to satisfy

$$
F_{1}=F_{0}+a \otimes n
$$

for some $a, n \in \mathbb{R}^{3},|n|=1$. By Lemma 2.1, we may assume without loss of generality that $F_{0} \in \mathcal{U}_{1}$ and $F_{1} \in \mathcal{U}_{2}$ and also that

$$
n=e_{1}
$$

for the orthorhombic to monoclinic transformation and

$$
n=\frac{1}{\sqrt{2}}\left(e_{1}+e_{2}\right)
$$

for the cubic to tetragonal transformation. Let $\lambda$ be a constant such that $0<\lambda<1$ and let

$$
F_{\lambda}=(1-\lambda) F_{0}+\lambda F_{1} .
$$

We define the set of admissible deformations which are compatible with the simple laminate to be

$$
W_{\lambda}^{1, \infty}\left(\Omega ; \mathbb{R}^{3}\right) \equiv\left\{y \in W^{1, \infty}\left(\Omega ; \mathbb{R}^{3}\right): y(x)=F_{\lambda} x, \forall x \in \partial \Omega\right\} .
$$

Our multi-well energy minimization problem is to minimize the elastic energy (2.1) among all deformations $y \in W_{\lambda}^{1, \infty}\left(\Omega ; \mathbb{R}^{3}\right)$. Ball and James have shown that there exist no energy minimizers for this minimization problem and that any energy minimizing sequence will converge to a unique microstructure which is composed of the gradient $F_{0}$ with volume fraction $1-\lambda$ and the gradient $F_{1}$ with volume fraction $\lambda[4]$.

We note that the proofs given in this paper for the orthorhombic to monoclinic transformation hold without modification for the more general problem with a rotationally invariant, double well energy (that is, $N=2$, in (1.1)) if there exists a rotation $Q \in \mathrm{SO}(3)$ and vectors $a, n \in \mathbb{R}^{3},|n|=1$, such that

$$
Q U_{2}=U_{1}+a \otimes n \text {. }
$$




\section{NONCONFORMING FINITE ELEMENTS}

We will denote a generic point in $\mathbb{R}^{3}$ by $\left(x_{1}, x_{2}, x_{3}\right)$. Our first finite element is defined by the triple $\left(\mathcal{Q}, P_{\mathcal{Q}}, \Sigma_{\mathcal{Q}}^{p}\right)$; where $\mathcal{Q} \equiv\left[\alpha_{1}-l_{1}, \alpha_{1}+l_{1}\right] \times\left[\alpha_{2}-l_{2}, \alpha_{2}+l_{2}\right] \times$ $\left[\alpha_{3}-l_{3}, \alpha_{3}+l_{3}\right]$ is a rectangular parallelepiped with its center at $\left(\alpha_{1}, \alpha_{2}, \alpha_{3}\right)$ and the lengths of its edges are $2 l_{1}, 2 l_{2}$, and $2 l_{3}$, where $l_{1}, l_{2}, l_{3}>0$;

$$
P_{\mathcal{Q}}=\operatorname{span}\left\{1, x_{1}, x_{2}, x_{3},\left(\frac{x_{1}}{l_{1}}\right)^{2}-\left(\frac{x_{2}}{l_{2}}\right)^{2},\left(\frac{x_{1}}{l_{1}}\right)^{2}-\left(\frac{x_{3}}{l_{3}}\right)^{2}\right\}
$$

and the set of degrees of freedom $\Sigma_{\mathcal{Q}}^{p}$ (the superscript $p$ denotes point) are given by

$$
\Sigma_{\mathcal{Q}}^{p}=\left\{q\left(c_{\mathcal{F}_{i}}\right): i=1, \cdots, 6\right\},
$$

where $c_{\mathcal{F}_{i}}, i=1, \cdots, 6$, are the centers of the faces $\mathcal{F}_{i}, i=1, \cdots, 6$, of the rectangular parallelepiped $\mathcal{Q}$. Our second element is defined to be the triple $\left(\mathcal{Q}, P_{\mathcal{Q}}, \Sigma_{\mathcal{Q}}^{a}\right)$. The polynomial space $P_{\mathcal{Q}}$ is the same as defined in (3.1) and the set of degrees of freedom $\Sigma_{\mathcal{Q}}^{a}$ (the superscript a denotes average) is defined by

$$
\Sigma_{\mathcal{Q}}^{a}=\left\{\frac{1}{\left|\mathcal{F}_{i}\right|} \int_{\mathcal{F}_{i}} q d S: i=1, \cdots, 6\right\}
$$

where $\mathcal{F}_{i}, i=1, \cdots, 6$, are the faces of $\mathcal{Q}$, and $\left|\mathcal{F}_{i}\right|$ is the area of the face $\mathcal{F}_{i}$ for $i=1, \cdots, 6$.

In the sequel, we will restrict ourselves to considering rectangular domains with faces parallel to coordinate planes. The results presented in this paper can be immediately extended to domains which are the union of rectangular parallelepipeds. However, we will assume for simplicity of exposition that $\Omega=\left(0, L_{1}\right) \times\left(0, L_{2}\right) \times$ $\left(0, L_{3}\right)$ for some $L_{k}>0, k=1,2,3$. To construct a rectangular partition $\tau_{h}$ of $\Omega$, we define the one-dimensional partitions of $\left[0, L_{k}\right]$, for $k=1,2,3$, by

$$
0=x_{k}^{0}<x_{k}^{1}<\cdots<x_{k}^{m_{k}}=L_{k},
$$

where the $m_{k}$ are positive integers. We then define the rectangular parallelepipeds

$$
\mathcal{R}_{i_{1}, i_{2}, i_{3}} \equiv\left[x_{1}^{i_{1}-1}, x_{1}^{i_{1}}\right] \times\left[x_{2}^{i_{2}-1}, x_{2}^{i_{2}}\right] \times\left[x_{3}^{i_{3}-1}, x_{3}^{i_{3}}\right]
$$

for $1 \leq i_{1} \leq m_{1}, 1 \leq i_{2} \leq m_{2}, 1 \leq i_{3} \leq m_{3}$, and the rectangular partition

$$
\tau_{h} \equiv\left\{\mathcal{R}_{i_{1}, i_{2}, i_{3}}: 1 \leq i_{1} \leq m_{1}, 1 \leq i_{2} \leq m_{2}, 1 \leq i_{3} \leq m_{3}\right\}
$$

The mesh size parameter $h$ is defined by $h=\max \left\{h_{k}: 1 \leq k \leq 3\right\}$, where $h_{k} \equiv$ $\max \left\{x_{k}^{i}-x_{k}^{i-1}: 1 \leq i \leq m_{k}\right\}$ is the maximal discretization size in the $k$ th coordinate direction for $k=1,2,3$. We will always assume that the rectangular partitions $\tau_{h}$ are quasi-uniform, that is, there exists a constant $\sigma>0$, independent of $h$, such that

$$
\min \left\{x_{k}^{i}-x_{k}^{i-1}: i=1, \ldots, m_{k}, k=1,2,3\right\} \geq \sigma h .
$$

For the first finite element, we define the set of nodal points $N_{h}$ to be the set of all centers $c_{\mathcal{F}}$ of faces $\mathcal{F}$ of elements in $\tau_{h}$. The finite element spaces over the 
partition $\tau_{h}$ are then defined respectively to be

$$
\begin{aligned}
& V_{h}^{p} \equiv\left\{v_{h} \in L^{2}(\Omega):\left.v_{h}\right|_{\mathcal{R}} \in P_{\mathcal{R}}, \forall \mathcal{R} \in \tau_{h} ; \text { adjoining } v_{h}\right. \text { have the same } \\
& \left.\quad \text { values at shared nodal points, that is, } v_{h} \text { is continuous on } N_{h}\right\}, \\
& V_{h}^{a} \equiv\left\{v_{h} \in L^{2}(\Omega):\left.v_{h}\right|_{\mathcal{R}} \in P_{\mathcal{R}}, \forall \mathcal{R} \in \tau_{h} ;\right. \\
& \left.\left.\quad \int_{\mathcal{F}} v_{h}\right|_{\mathcal{R}^{\prime}} d S=\left.\int_{\mathcal{F}} v_{h}\right|_{\mathcal{R}^{\prime \prime}} d S, \forall \text { faces } \mathcal{F}=\partial \mathcal{R}^{\prime} \cap \partial \mathcal{R}^{\prime \prime} \neq \emptyset, \mathcal{R}^{\prime}, \mathcal{R}^{\prime \prime} \in \tau_{h}\right\} .
\end{aligned}
$$

We denote by $\mathcal{A}_{h}^{p}$ the set of admissible finite element deformations $y_{h}: \Omega \rightarrow \mathbb{R}^{3}$ such that each component of $y_{h}$ belongs to $V_{h}^{p}$ and such that $y_{h}\left(c_{\mathcal{F}}\right)=F_{\lambda} c_{\mathcal{F}}$ if $c_{\mathcal{F}}$ is the center of an element face $\mathcal{F}$ lying in $\partial \Omega$. Similarly, we denote $\mathcal{A}_{h}^{a}$ to be the set of admissible finite element deformations $y_{h}: \Omega \rightarrow \mathbb{R}^{3}$ such that each component of $y_{h}$ belongs to $V_{h}^{a}$ and such that

$$
\int_{\mathcal{F}} y_{h}(x) d S=\int_{\mathcal{F}} F_{\lambda} x d S
$$

for any element face $\mathcal{F} \subset \partial \Omega$. Note that the deformation $y_{h}(x)=F_{\lambda} x, x \in \Omega$, belongs to both $\mathcal{A}_{h}^{p}$ and $\mathcal{A}_{h}^{a}$. We denote for convenience $V_{h}=V_{h}^{p} \cup V_{h}^{a}$ and $\mathcal{A}_{h}=$ $\mathcal{A}_{h}^{p} \cup \mathcal{A}_{h}^{a}$.

It is obvious that both of the spaces $V_{h}^{p}$ and $V_{h}^{a}$ are finite-dimensional affine subspaces of $L^{2}(\Omega)$. They are also affine finite element spaces [10]. For $v_{h} \in V_{h}^{p}$ or $v_{h} \in V_{h}^{a}$, we have in general that $v_{h} \notin C(\bar{\Omega})$ since $v_{h}$ is continuous only at some points of the faces of adjacent elements. Thus, $V_{h}^{p}, V_{h}^{a} \nsubseteq C(\bar{\Omega})$, and hence, neither $\mathcal{A}_{h}^{p}$ nor $\mathcal{A}_{h}^{a}$ is contained in $W_{\lambda}^{1, \infty}\left(\Omega ; \mathbb{R}^{3}\right)$ which is a subset of $C\left(\bar{\Omega} ; \mathbb{R}^{3}\right)$ by the embedding theorem [1]. Therefore, in view of minimizing the elastic energy over $W_{\lambda}^{1, \infty}\left(\Omega ; \mathbb{R}^{3}\right) \subset W^{1, \infty}\left(\Omega ; \mathbb{R}^{3}\right)$, the above finite elements are nonconforming.

We now denote the Lagrange interpolation operator $I_{h}: C(\bar{\Omega}) \rightarrow V_{h}$ to be either $I_{h}^{p}: C(\bar{\Omega}) \rightarrow V_{h}^{p}$ or $I_{h}^{a}: C(\bar{\Omega}) \rightarrow V_{h}^{a}$, which are defined respectively by $I_{h}^{p} v \in V_{h}^{p}$ and $I_{h}^{a} v \in V_{h}^{a}$, and

$$
\begin{array}{ll}
I_{h}^{p} v\left(c_{\mathcal{F}}\right)=v\left(c_{\mathcal{F}}\right), & \forall c_{F} \in N_{h}, \\
\int_{\mathcal{F}} I_{h}^{a} v d S=\int_{\mathcal{F}} v d S, \quad \forall \text { faces } \mathcal{F} \subset \partial \mathcal{R}, \forall \mathcal{R} \in \tau_{h},
\end{array}
$$

for any $v \in C(\bar{\Omega})$. We will also use the same notation $I_{h}, I_{h}^{p}$ and $I_{h}^{a}$ to denote the restrictions of these operators to an element of the partition $\tau_{h}$.

For any element $\mathcal{R} \in \tau_{h}$ and a face $\mathcal{F} \subset \partial \mathcal{R}$, we define the functional $T_{\mathcal{F}}^{p}$ : $C(\mathcal{F}) \rightarrow \mathbb{R}$ by $T_{\mathcal{F}}^{p}(w)=w\left(c_{\mathcal{F}}\right)$ for $w \in C(\mathcal{F})$, where $c_{\mathcal{F}}$ is the center of the face $\mathcal{F}$, when considering the $V_{h}^{p}$-approximation, and the functional $T_{\mathcal{F}}^{a}: L^{2}(\mathcal{F}) \rightarrow \mathbb{R}$ by $T_{\mathcal{F}}^{a}(w)=(1 /|\mathcal{F}|) \int_{\mathcal{F}} w d S$ for $w \in L^{2}(\mathcal{F})$, when considering the $V_{h}^{a}$-approximation. Similar functionals of suitable deformations can be defined component-wise. Without confusion, the same notation $T_{\mathcal{F}}^{p}$ or $T_{\mathcal{F}}^{a}$ will be used for functionals defined on both scalar functions and vectorial deformations.

We will use the letter $C$ to denote a generic positive constant which is independent of the mesh size $h$. For convenience, we also define for any integer $k \geq 0$ and $p \in[1, \infty]$ the space

$$
W_{h}^{k, p}(\Omega) \equiv\left\{v \in L^{p}(\Omega):\left.v\right|_{\mathcal{R}} \in W^{k, p}(\mathcal{R}), \forall \mathcal{R} \in \tau_{h}\right\},
$$


and we equip $W_{h}^{k, p}(\Omega)$ with the following semi-norm and norm:

$$
\begin{aligned}
|\cdot|_{k, p, h} & \equiv \begin{cases}\left(\sum|\cdot|_{k, p, \mathcal{R}}^{p}\right)^{\frac{1}{p}}, & \text { if } 1 \leq p<\infty, \\
\max _{\mathcal{R} \in \tau_{h}}|\cdot|_{k, \infty, \mathcal{R}}, & \text { if } p=\infty ;\end{cases} \\
\|\cdot\|_{k, p, h} & \equiv \begin{cases}\left(\sum\|\cdot\|_{k, p, \mathcal{R}}^{p}\right)^{\frac{1}{p}}, & \text { if } 1 \leq p<\infty, \\
\max _{\mathcal{R} \in \tau_{h}}\|\cdot\|_{k, \infty, \mathcal{R}}, & \text { if } p=\infty ;\end{cases}
\end{aligned}
$$

where, for $\mathcal{R} \in \tau_{h},|\cdot|_{k, p, \mathcal{R}}$ and $\|\cdot\|_{k, p, \mathcal{R}}$ are the usual semi-norm and norm on the Sobolev space $W^{k, p}(\mathcal{R})$ [1]. If $p=2$ we write $H_{h}^{k}(\Omega)$ for $W_{h}^{k, p}(\Omega)$ and omit $p$ in all of the above semi-norm and norm expressions. We define the spaces $W_{h}^{k, p}\left(\Omega ; \mathbb{R}^{3}\right)$ and $H_{h}^{k}\left(\Omega ; \mathbb{R}^{3}\right)$ in a similar way and use the same notation $|\cdot|_{k, p, h},\|\cdot\|_{k, p, h},|\cdot|_{k, h}$, and $\|\cdot\|_{k, h}$ for the associated semi-norms and norms.

We now collect some useful properties of the finite element spaces $V_{h}^{p}$ and $V_{h}^{a}$ in the following lemmas.

Lemma 3.1. For any $v_{h} \in V_{h}=V_{h}^{p} \cup V_{h}^{a}$ restricted to any $\mathcal{R} \in \tau_{h}$, we have

$$
\frac{\partial v_{h}}{\partial x_{k}} \in \operatorname{span}\left\{1, x_{k}\right\}, \quad k=1,2,3 .
$$

It follows that

$$
\begin{aligned}
& v_{h}\left(\hat{x}_{1}, \hat{x}_{2}, \hat{x}_{3}\right)-v_{h}\left(\hat{x}_{1}, x_{2}, x_{3}\right)=v_{h}\left(x_{1}, \hat{x}_{2}, \hat{x}_{3}\right)-v_{h}\left(x_{1}, x_{2}, x_{3}\right), \\
& v_{h}\left(\hat{x}_{1}, \hat{x}_{2}, \hat{x}_{3}\right)-v_{h}\left(x_{1}, \hat{x}_{2}, x_{3}\right)=v_{h}\left(\hat{x}_{1}, x_{2}, \hat{x}_{3}\right)-v_{h}\left(x_{1}, x_{2}, x_{3}\right), \\
& v_{h}\left(\hat{x}_{1}, \hat{x}_{2}, \hat{x}_{3}\right)-v_{h}\left(x_{1}, x_{2}, \hat{x}_{3}\right)=v_{h}\left(\hat{x}_{1}, \hat{x}_{2}, x_{3}\right)-v_{h}\left(x_{1}, x_{2}, x_{3}\right) .
\end{aligned}
$$

for any $\left(\hat{x}_{1}, \hat{x}_{2}, \hat{x}_{3}\right) \in \mathcal{R}$ and $\left(x_{1}, x_{2}, x_{3}\right) \in \mathcal{R}$.

Proof. The equation (3.3) follows directly from the definition of the finite element polynomial space $P_{\mathcal{Q}}$ (3.1). The result (3.4) follows from (3.3) since $\partial v_{h} / \partial x_{1}$ is independent of $x_{2}$ and $x_{3}$, so

$$
\begin{aligned}
v_{h}\left(\hat{x}_{1}, \hat{x}_{2}, \hat{x}_{3}\right)-v_{h}\left(x_{1}, \hat{x}_{2}, \hat{x}_{3}\right) & =\int_{x_{1}}^{\hat{x}_{1}} \frac{\partial v_{h}}{\partial x_{1}}\left(\xi, \hat{x}_{2}, \hat{x}_{3}\right) d \xi \\
& =\int_{x_{1}}^{\hat{x}_{1}} \frac{\partial v_{h}}{\partial x_{1}}\left(\xi, x_{2}, x_{3}\right) d \xi \\
& =v_{h}\left(\hat{x}_{1}, x_{2}, x_{3}\right)-v_{h}\left(x_{1}, x_{2}, x_{3}\right) .
\end{aligned}
$$

The results (3.5) and (3.6) follow similarly.

Lemma 3.2. Let $k$ and $l$ be two integers such that $0 \leq k \leq l \leq 2$. We have the following inverse inequalities for any $\mathcal{R} \in \tau_{h}$ and any $v_{h} \in V_{h}=V_{h}^{p} \cup V_{h}^{a}$ :

$$
\begin{aligned}
& \left|v_{h}\right|_{l, \mathcal{R}} \leq C h^{k-l}\left|v_{h}\right|_{k, \mathcal{R}}, \\
& \left|v_{h}\right|_{l, h} \leq C h^{k-l}\left|v_{h}\right|_{k, h}, \\
& \left|v_{h}\right|_{l, \infty, \mathcal{R}} \leq C h^{k-l-\frac{3}{2}}\left|v_{h}\right|_{k, \mathcal{R}}, \\
& \left|v_{h}\right|_{l, \infty, h} \leq C h^{k-l-\frac{3}{2}}\left|v_{h}\right|_{k, h} .
\end{aligned}
$$

Proof. Since both $V_{h}^{p}$ and $V_{h}^{a}$ are affine finite element spaces, the results of this lemma can be proven by a standard argument via affine mappings [10]. 
Lemma 3.3. We have for any $\mathcal{R} \in \tau_{h}$ and any face $\mathcal{F} \subset \partial \mathcal{R}$ that

$$
\int_{\mathcal{F}}\left|v-T_{\mathcal{F}}^{a}(v)\right|^{2} d S \leq C h|v|_{1, \mathcal{R}}^{2}, \quad \forall v \in H^{1}(\mathcal{R}) .
$$

We also have that

$$
\int_{\mathcal{F}}\left|v_{h}-T_{\mathcal{F}}^{p}\left(v_{h}\right)\right|^{2} d S \leq C h\left|v_{h}\right|_{1, \mathcal{R}}^{2}, \quad \forall v_{h} \in V_{h}^{p} .
$$

Proof. We will prove (3.11) and (3.12) on the reference domain $\hat{\mathcal{R}}=(0,1) \times(0,1) \times$ $(0,1)$ with face $\hat{\mathcal{F}}=\{0\} \times(0,1) \times(0,1)$. We can then obtain the results $(3.11)$ and (3.12) on the element $\mathcal{R} \in \tau_{h}$ and the face $\mathcal{F} \subset \partial \mathcal{R}$ by an affine scaling.

For $v \in C^{\infty}(\hat{\mathcal{R}})$ we have that

$$
\begin{aligned}
v\left(0, x_{2}, x_{3}\right)- & \int_{0}^{1} \int_{0}^{1} v\left(0, \hat{x}_{2}, \hat{x}_{3}\right) d \hat{x}_{2} d \hat{x}_{3} \\
= & v\left(x_{1}, x_{2}, x_{3}\right)-\int_{0}^{1} \int_{0}^{1} v\left(x_{1}, \hat{x}_{2}, \hat{x}_{3}\right) d \hat{x}_{2} d \hat{x}_{3} \\
& \quad-\int_{0}^{x_{1}} \frac{\partial v}{\partial x_{1}}\left(\hat{x}_{1}, x_{2}, x_{3}\right) d \hat{x}_{1}+\int_{0}^{1} \int_{0}^{1} \int_{0}^{x_{1}} \frac{\partial v}{\partial x_{1}}\left(\hat{x}_{1}, \hat{x}_{2}, \hat{x}_{3}\right) d \hat{x}_{1} d \hat{x}_{2} d \hat{x}_{3} \\
= & \int_{0}^{1} \int_{0}^{1}\left[v\left(x_{1}, x_{2}, x_{3}\right)-v\left(x_{1}, \hat{x}_{2}, \hat{x}_{3}\right)\right] d \hat{x}_{2} d \hat{x}_{3} \\
& \quad-\int_{0}^{x_{1}} \frac{\partial v}{\partial x_{1}}\left(\hat{x}_{1}, x_{2}, x_{3}\right) d \hat{x}_{1}+\int_{0}^{1} \int_{0}^{1} \int_{0}^{x_{1}} \frac{\partial v}{\partial x_{1}}\left(\hat{x}_{1}, \hat{x}_{2}, \hat{x}_{3}\right) d \hat{x}_{1} d \hat{x}_{2} d \hat{x}_{3} .
\end{aligned}
$$

Now,

$$
\begin{aligned}
v\left(x_{1},\right. & \left.x_{2}, x_{3}\right)-v\left(x_{1}, \hat{x}_{2}, \hat{x}_{3}\right) \\
& =\left[v\left(x_{1}, x_{2}, x_{3}\right)-v\left(x_{1}, \hat{x}_{2}, x_{3}\right)\right]+\left[v\left(x_{1}, \hat{x}_{2}, x_{3}\right)-v\left(x_{1}, \hat{x}_{2}, \hat{x}_{3}\right)\right] \\
& =\int_{\hat{x}_{2}}^{x_{2}} \frac{\partial v}{\partial x_{2}}\left(x_{1}, \check{x}_{2}, x_{3}\right) d \check{x}_{2}+\int_{\hat{x}_{3}}^{x_{3}} \frac{\partial v}{\partial x_{3}}\left(x_{1}, \hat{x}_{2}, \check{x}_{3}\right) d \check{x}_{3} .
\end{aligned}
$$

We obtain from substituting (3.14) into (3.13) that

$$
\begin{aligned}
v\left(0, x_{2}, x_{3}\right)- & \int_{0}^{1} \int_{0}^{1} v\left(0, \hat{x}_{2}, \hat{x}_{3}\right) d \hat{x}_{2} d \hat{x}_{3} \\
= & \int_{0}^{1} \int_{0}^{1} \int_{\hat{x}_{2}}^{x_{2}} \frac{\partial v}{\partial x_{2}}\left(x_{1}, \check{x}_{2}, x_{3}\right) d \check{x}_{2} d \hat{x}_{2} d \hat{x}_{3} \\
& +\int_{0}^{1} \int_{0}^{1} \int_{\hat{x}_{3}}^{x_{3}} \frac{\partial v}{\partial x_{3}}\left(x_{1}, \hat{x}_{2}, \check{x}_{3}\right) d \check{x}_{3} d \hat{x}_{2} d \hat{x}_{3} \\
& \quad-\int_{0}^{x_{1}} \frac{\partial v}{\partial x_{1}}\left(\hat{x}_{1}, x_{2}, x_{3}\right) d \hat{x}_{1}+\int_{0}^{1} \int_{0}^{1} \int_{0}^{x_{1}} \frac{\partial v}{\partial x_{1}}\left(\hat{x}_{1}, \hat{x}_{2}, \hat{x}_{3}\right) d \hat{x}_{1} d \hat{x}_{2} d \hat{x}_{3} .
\end{aligned}
$$

We can then obtain by squaring both sides of (3.15), integrating with respect to $\left(x_{1}, x_{2}, x_{3}\right)$ over the domain $(0,1) \times(0,1) \times(0,1)$, and using the Cauchy-Schwarz 
inequality that

$$
\begin{aligned}
\int_{0}^{1} & \int_{0}^{1}\left|v\left(0, x_{2}, x_{3}\right)-\int_{0}^{1} \int_{0}^{1} v\left(0, \hat{x}_{2}, \hat{x}_{3}\right) d \hat{x}_{2} d \hat{x}_{3}\right|^{2} d x_{2} d x_{3} \\
& =\int_{0}^{1} \int_{0}^{1} \int_{0}^{1}\left|v\left(0, x_{2}, x_{3}\right)-\int_{0}^{1} \int_{0}^{1} v\left(0, \hat{x}_{2}, \hat{x}_{3}\right) d \hat{x}_{2} d \hat{x}_{3}\right|^{2} d x_{1} d x_{2} d x_{3} \\
& \leq 8\left|\frac{\partial v}{\partial x_{1}}\right|_{0, \hat{\mathcal{R}}}^{2}+4\left|\frac{\partial v}{\partial x_{2}}\right|_{0, \hat{\mathcal{R}}}^{2}+4\left|\frac{\partial v}{\partial x_{3}}\right|_{0, \hat{\mathcal{R}}}^{2} .
\end{aligned}
$$

The inequality (3.11) for $\hat{\mathcal{R}}$ and $\hat{\mathcal{F}}$ now follows from the density of $C^{\infty}(\hat{\mathcal{R}})$ in $H^{1}(\hat{\mathcal{R}})$ and the continuous embedding $H^{1}(\hat{\mathcal{R}}) \hookrightarrow L^{2}(\hat{\mathcal{F}})[1]$.

We note that we cannot prove the inequality (3.12) for all $v \in H^{1}(\mathcal{R})$ because $T_{\mathcal{F}}^{p}(v)=v\left(c_{\mathcal{F}}\right)$ is not a well-defined operator on $H^{1}(\mathcal{R})$ since $H^{1}(\hat{\mathcal{R}})$ is not continuously embedded in $C(\hat{\mathcal{R}})$ [1]. To prove the inequality (3.12) with $\mathcal{R}$ and $\mathcal{F}$ replaced by $\hat{\mathcal{R}}$ and $\hat{\mathcal{F}}$, respectively, for $v_{h} \in P_{\hat{\mathcal{R}}}$, the finite element polynomial space (3.1), we derive as above the identity

$$
\begin{aligned}
& v_{h}\left(0, x_{2}, x_{3}\right)-v_{h}(0,1 / 2,1 / 2) \\
& =\int_{1 / 2}^{x_{2}} \frac{\partial v_{h}}{\partial x_{2}}\left(x_{1}, \check{x}_{2}, x_{3}\right) d \check{x}_{2}+\int_{1 / 2}^{x_{3}} \frac{\partial v_{h}}{\partial x_{3}}\left(x_{1}, 1 / 2, \check{x}_{3}\right) d \check{x}_{3} \\
& \quad-\int_{0}^{x_{1}} \frac{\partial v_{h}}{\partial x_{1}}\left(\hat{x}_{1}, x_{2}, x_{3}\right) d \hat{x}_{1}+\int_{0}^{x_{1}} \frac{\partial v_{h}}{\partial x_{1}}\left(\hat{x}_{1}, 1 / 2,1 / 2\right) d \hat{x}_{1} .
\end{aligned}
$$

Since by Lemma $3.1, \partial v_{h} / \partial x_{k} \in \operatorname{span}\left\{1, x_{k}\right\}$, for $k=1,2,3$, we have from (3.17) that

$$
\begin{aligned}
& v_{h}\left(0, x_{2}, x_{3}\right)-v_{h}(0,1 / 2,1 / 2) \\
& =\int_{1 / 2}^{x_{2}} \frac{\partial v_{h}}{\partial x_{2}}\left(x_{1}, \check{x}_{2}, x_{3}\right) d \check{x}_{2}+\int_{1 / 2}^{x_{3}} \frac{\partial v_{h}}{\partial x_{3}}\left(x_{1}, x_{2}, \check{x}_{3}\right) d \check{x}_{3} \\
& \quad-\int_{0}^{x_{1}} \frac{\partial v_{h}}{\partial x_{1}}\left(\hat{x}_{1}, x_{2}, x_{3}\right) d \hat{x}_{1}+\int_{0}^{x_{1}} \frac{\partial v_{h}}{\partial x_{1}}\left(\hat{x}_{1}, x_{2}, x_{3}\right) d \hat{x}_{1} .
\end{aligned}
$$

We can then obtain by squaring both sides of (3.18), integrating with respect to $\left(x_{1}, x_{2}, x_{3}\right)$ over the domain $(0,1) \times(0,1) \times(0,1)$, and using the Cauchy-Schwarz inequality that

$$
\begin{gathered}
\int_{0}^{1} \int_{0}^{1}\left|v_{h}\left(0, x_{2}, x_{3}\right)-v_{h}(0,1 / 2,1 / 2)\right|^{2} d x_{2} d x_{3} \\
\quad \leq 8\left|\frac{\partial v_{h}}{\partial x_{1}}\right|_{0, \hat{\mathcal{R}}}^{2}+4\left|\frac{\partial v_{h}}{\partial x_{2}}\right|_{0, \hat{\mathcal{R}}}^{2}+4\left|\frac{\partial v_{h}}{\partial x_{3}}\right|_{0, \hat{\mathcal{R}}}^{2} .
\end{gathered}
$$

Lemma 3.4. We have

$$
\left\|\nabla I_{h} v\right\|_{0, \infty, h} \leq C\|\nabla v\|_{0, \infty, \Omega}, \quad \forall v \in W^{1, \infty}(\Omega) .
$$

Proof. The proof easily follows from the quasi-uniformity of the partition $\tau_{h}$ [10]. 


\section{Properties of NONCONFORMing Finite ELEMENT DEFORMATIONS}

In this section, we will give some further properties of the considered nonconforming finite element deformations. We first prove a discrete version of a slight variation of the divergence theorem.

Theorem 4.1. We have for any $y_{h} \in \mathcal{A}_{h}=\mathcal{A}_{h}^{p} \cup \mathcal{A}_{h}^{a}$ that

$$
\sum_{\mathcal{R} \in \tau_{h}} \int_{\mathcal{R}} \nabla y_{h}(x) d x=\sum_{\mathcal{R} \in \tau_{h}} \int_{\mathcal{R}} F_{\lambda} d x
$$

Proof. Applying the divergence theorem to each integral on $\mathcal{R} \in \tau_{h}$ in the summation and noticing the cancellation of contributions from adjacent elements to their common faces, we see by the definition of $\mathcal{A}_{h}^{a}$ that (4.1) holds if $y_{h} \in \mathcal{A}_{h}^{a}$.

For $y_{h} \in \mathcal{A}_{h}^{p}$, we set $z_{h}(x)=y_{h}(x)-F_{\lambda} x, x \in \Omega$. We also denote by $c_{\mathcal{F}}$ the center of a face $\mathcal{F}$ of an element in $\tau_{h}$. Thus, by the definition of $\mathcal{A}_{h}^{p}$, we have $z_{h}\left(c_{\mathcal{F}}\right)=0$ if $\mathcal{F} \subset \partial \Omega$. Moreover, we have

$$
\begin{aligned}
\sum_{\mathcal{R} \in \tau_{h}} \int_{\mathcal{R}} \nabla z_{h}(x) d x=\sum_{\mathcal{R} \in \tau_{h}} \int_{\partial \mathcal{R}} z_{h}(x) \otimes \nu d S \\
=\left.\sum_{\mathcal{R} \in \tau_{h}} \sum_{\mathcal{F} \subset \partial \mathcal{R}} \int_{\mathcal{F}}\left[z_{h}(x)-z_{h}\left(c_{\mathcal{F}}\right)\right] \otimes \nu\right|_{\mathcal{F}} d S,
\end{aligned}
$$

where $\nu$ is the unit exterior normal to the underlying boundary.

Fix $\mathcal{R}=\left[\alpha_{1}-l_{1}, \alpha_{1}+l_{1}\right] \times\left[\alpha_{2}-l_{2}, \alpha_{2}+l_{2}\right] \times\left[\alpha_{3}-l_{3}, \alpha_{3}+l_{3}\right] \in \tau_{h}$. Set $\mathcal{F}_{ \pm}=\left[\alpha_{1}-l_{1}, \alpha_{1}+l_{1}\right] \times\left[\alpha_{2}-l_{2}, \alpha_{2}+l_{2}\right] \times\left\{\alpha_{3} \pm l_{3}\right\}$. It follows from (3.6) that

$z_{h}\left(x_{1}, x_{2}, \alpha_{3}+l_{3}\right)-z_{h}\left(\alpha_{1}, \alpha_{2}, \alpha_{3}+l_{3}\right)=z_{h}\left(x_{1}, x_{2}, \alpha_{3}-l_{3}\right)-z_{h}\left(\alpha_{1}, \alpha_{2}, \alpha_{3}-l_{3}\right)$.

Noting that $\left.\nu\right|_{\mathcal{F}_{+}}=-\left.\nu\right|_{\mathcal{F}_{-}}=e_{3}$, we then have

$$
\begin{aligned}
\int_{\mathcal{F}_{+}} & {\left.\left[z_{h}(x)-z_{h}\left(c_{\mathcal{F}_{+}}\right)\right] \otimes \nu\right|_{\mathcal{F}_{+}} d S+\left.\int_{\mathcal{F}_{-}}\left[z_{h}(x)-z_{h}\left(c_{\mathcal{F}_{-}}\right)\right] \otimes \nu\right|_{\mathcal{F}_{-}} d S } \\
= & {\left[\int _ { \alpha _ { 1 } - l _ { 1 } } ^ { \alpha _ { 1 } + l _ { 1 } } \int _ { \alpha _ { 2 } - l _ { 2 } } ^ { \alpha _ { 2 } + l _ { 2 } } \left\{\left[z_{h}\left(x_{1}, x_{2}, \alpha_{3}+l_{3}\right)-z_{h}\left(\alpha_{1}, \alpha_{2}, \alpha_{3}+l_{3}\right)\right]\right.\right.} \\
& \left.\left.\quad-\left[z_{h}\left(x_{1}, x_{2}, \alpha_{3}-l_{3}\right)-z_{h}\left(\alpha_{1}, \alpha_{2}, \alpha_{3}-l_{3}\right)\right]\right\} d x_{1} d x_{2}\right] \otimes e_{3} \\
=0 . &
\end{aligned}
$$

The same argument applies to any other pair of faces $\mathcal{F}_{ \pm} \subset \partial \mathcal{R}$. Therefore,

$$
\left.\sum_{\mathcal{F} \subset \partial \mathcal{R}} \int_{\mathcal{F}}\left[z_{h}(x)-z_{h}\left(c_{\mathcal{F}}\right)\right] \otimes \nu\right|_{\mathcal{F}} d S=0 .
$$

The arbitrariness of $\mathcal{R} \in \tau_{h}$ then implies that the sum in (4.2) is zero. This proves (4.1) for $y_{h} \in \mathcal{A}_{h}^{p}$ as well.

We now prove a Poincaré type inequality for all of the finite element deformations in $\mathcal{A}_{h}$. This result is more general than that proven in [24]. 
Theorem 4.2. There exists a constant $C>0$ such that for all $w \in \mathbb{R}^{3}$ with $|w|=1$ and all $y_{h} \in \mathcal{A}_{h}$,

$$
\begin{aligned}
& \int_{\Omega}\left|y_{h}(x)-F_{\lambda} x\right|^{2} d x \\
& \quad \leq C \sum_{\mathcal{R} \in \tau_{h}} \int_{\mathcal{R}}\left\{\left|\left[\nabla y_{h}(x)-F_{\lambda}\right] w\right|^{2}+h\left\|\nabla y_{h}(x)-F_{\lambda}\right\|^{2}\right\} d x .
\end{aligned}
$$

Proof. Fix an arbitrary $w \in \mathbb{R}^{3}$ with $|w|=1$. For $y_{h} \in \mathcal{A}_{h}$, set again $z_{h}(x)=$ $y_{h}(x)-F_{\lambda} x, x \in \Omega$. By integration by parts we obtain [44]

$$
\begin{aligned}
\int_{\Omega}\left|z_{h}(x)\right|^{2} d x= & \sum_{\mathcal{R} \in \tau_{h}} \int_{\partial \mathcal{R}}\left|z_{h}(x)\right|^{2}(w \cdot x)(w \cdot \nu) d S \\
& \quad-\sum_{\mathcal{R} \in \tau_{h}} \int_{\mathcal{R}}\left(\nabla\left|z_{h}(x)\right|^{2} \cdot w\right)(w \cdot x) d x \\
& \equiv I_{1}+I_{2} .
\end{aligned}
$$

We estimate the second term $I_{2}$ by the Cauchy-Schwarz inequality to get

$$
\begin{aligned}
\left|I_{2}\right| & \equiv\left|\sum_{\mathcal{R} \in \tau_{h}} \int_{\mathcal{R}}\left(\nabla\left|z_{h}(x)\right|^{2} \cdot w\right)(w \cdot x) d x\right| \\
& \leq 2 \max _{x \in \bar{\Omega}}|w \cdot x|\left(\sum_{\mathcal{R} \in \tau_{h}} \int_{\mathcal{R}}\left|\nabla z_{h}(x) w\right|^{2} d x\right)^{\frac{1}{2}}\left(\int_{\Omega}\left|z_{h}(x)\right|^{2} d x\right)^{\frac{1}{2}} \\
& \leq \frac{1}{2} \int_{\Omega}\left|z_{h}(x)\right|^{2} d x+C \sum_{\mathcal{R} \in \tau_{h}} \int_{\mathcal{R}}\left|\nabla z_{h}(x) w\right|^{2} d x .
\end{aligned}
$$

To estimate $I_{1}$, we first consider the $\mathcal{A}_{h}^{a}$-approximation. So, we fix $y_{h} \in \mathcal{A}_{h}^{a}$. Observing that $T_{\mathcal{F}}^{a}\left(z_{h}\right)=0$ for any element face $\mathcal{F} \subset \partial \Omega$, we obtain by the definition of $\mathcal{A}_{h}^{a}$ that

$$
\begin{aligned}
I_{1} \equiv & \sum_{\mathcal{R} \in \tau_{h}} \int_{\partial \mathcal{R}}\left|z_{h}(x)\right|^{2}(w \cdot x)(w \cdot \nu) d S \\
= & \sum_{\mathcal{R} \in \tau_{h}} \sum_{\mathcal{F} \subset \partial \mathcal{R}} \int_{\mathcal{F}}\left|\left[z_{h}(x)-T_{\mathcal{F}}^{a}\left(z_{h}\right)\right]+T_{\mathcal{F}}^{a}\left(z_{h}\right)\right|^{2}(w \cdot x)\left(\left.w \cdot \nu\right|_{\mathcal{F}}\right) d S \\
= & \sum_{\mathcal{R} \in \tau_{h}} \sum_{\mathcal{F} \subset \partial \mathcal{R}} \int_{\mathcal{F}}\left|z_{h}(x)-T_{\mathcal{F}}^{a}\left(z_{h}\right)\right|^{2}(w \cdot x)\left(\left.w \cdot \nu\right|_{\mathcal{F}}\right) d S \\
& \quad+\sum_{\mathcal{R} \in \tau_{h}} \sum_{\mathcal{F} \subset \partial \mathcal{R}} \int_{\mathcal{F}}\left|T_{\mathcal{F}}^{a}\left(z_{h}\right)\right|^{2}(w \cdot x)(w \cdot \nu \mid \mathcal{F}) d S \\
& \quad+\sum_{\mathcal{R} \in \tau_{h}} \sum_{\mathcal{F} \subset \partial \mathcal{R}} \int_{\mathcal{F}} 2 T_{\mathcal{F}}^{a}\left(z_{h}\right) \cdot\left[z_{h}(x)-T_{\mathcal{F}}^{a}\left(z_{h}\right)\right](w \cdot x)\left(\left.w \cdot \nu\right|_{\mathcal{F}}\right) d S \\
= & \sum_{\mathcal{R} \in \tau_{h}} \sum_{\mathcal{F} \subset \partial \mathcal{R}} \int_{\mathcal{F}}\left|z_{h}(x)-T_{\mathcal{F}}^{a}\left(z_{h}\right)\right|^{2}(w \cdot x)\left(\left.w \cdot \nu\right|_{\mathcal{F}}\right) d S \\
& \quad+2 \sum_{\mathcal{R} \in \tau_{h}} \sum_{\mathcal{F} \subset \partial \mathcal{R}} \int_{\mathcal{F}} T_{\mathcal{F}}^{a}\left(z_{h}\right) \cdot\left[z_{h}(x)-T_{\mathcal{F}}^{a}\left(z_{h}\right)\right](w \cdot x)\left(\left.w \cdot \nu\right|_{\mathcal{F}}\right) d S \\
\equiv & J_{1}^{a}+2 J_{2}^{a},
\end{aligned}
$$


where we combined adjacent elements and canceled their contributions to the common face to obtain that one summed term is equal to zero. It follows directly from (3.11) that

$$
\begin{aligned}
\left|J_{1}^{a}\right| & \equiv\left|\sum_{\mathcal{R} \in \tau_{h}} \sum_{\mathcal{F} \subset \partial \mathcal{R}} \int_{\mathcal{F}}\right| z_{h}(x)-\left.T_{\mathcal{F}}^{a}\left(z_{h}\right)\right|^{2}(w \cdot x)\left(\left.w \cdot \nu\right|_{\mathcal{F}}\right) d S \mid \\
& \leq C h\left\|\nabla z_{h}\right\|_{0, h}^{2} .
\end{aligned}
$$

Setting $g_{\nu}(x)=(w \cdot x)(w \cdot \nu)$, we have

$$
\begin{aligned}
J_{2}^{a} & \equiv \sum_{\mathcal{R} \in \tau_{h}} \sum_{\mathcal{F} \subset \partial \mathcal{R}} \int_{\mathcal{F}} T_{\mathcal{F}}^{a}\left(z_{h}\right) \cdot\left[z_{h}(x)-T_{\mathcal{F}}^{a}\left(z_{h}\right)\right] g_{\nu}(x) d S \\
& =\sum_{\mathcal{R} \in \tau_{h}} \sum_{\mathcal{F} \subset \partial \mathcal{R}} \int_{\mathcal{F}} T_{\mathcal{F}}^{a}\left(z_{h}\right) \cdot\left[z_{h}(x)-T_{\mathcal{F}}^{a}\left(z_{h}\right)\right]\left[g_{\nu}(x)-T_{\mathcal{F}}^{a}\left(g_{\nu}\right)\right] d S .
\end{aligned}
$$

For a fixed face $\mathcal{F} \subset \partial \mathcal{R}$ of some element $\mathcal{R} \in \tau_{h}$, we have by the inverse estimate (3.9) that

$$
\left|T_{\mathcal{F}}^{a}\left(z_{h}\right)\right| \leq\left\|z_{h}\right\|_{0, \infty, \mathcal{R}} \leq C h^{-\frac{3}{2}}\left\|z_{h}\right\|_{0, \mathcal{R}} .
$$

We also have by (3.11) that

$$
\int_{\mathcal{F}}\left|z_{h}(x)-T_{\mathcal{F}}^{a}\left(z_{h}\right)\right|^{2} d S \leq C h\left\|\nabla z_{h}\right\|_{0, \mathcal{R}}^{2}
$$

and

$$
\int_{\mathcal{F}}\left|g_{\nu}(x)-T_{\mathcal{F}}^{a}\left(g_{\nu}\right)\right|^{2} d S \leq C h\left\|\nabla g_{\nu}\right\|_{0, \mathcal{R}}^{2} \leq C h^{4} .
$$

Consequently,

$$
\begin{aligned}
\left|J_{2}^{a}\right| \leq & \sum_{\mathcal{R} \in \tau_{h}} \sum_{\mathcal{F} \subset \partial \mathcal{R}}\left|T_{\mathcal{F}}^{a}\left(z_{h}\right)\right|\left(\int_{\mathcal{F}}\left|z_{h}(x)-T_{\mathcal{F}}^{a}\left(z_{h}\right)\right|^{2} d S\right)^{\frac{1}{2}} \\
& \cdot\left(\int_{\mathcal{F}}\left|g_{\nu}(x)-T_{\mathcal{F}}^{a}\left(g_{\nu}\right)\right|^{2} d S\right)^{\frac{1}{2}} \\
\leq & C h \sum_{\mathcal{R} \in \tau_{h}}\left\|z_{h}\right\|_{0, \mathcal{R}}\left\|\nabla z_{h}\right\|_{0, \mathcal{R}} \\
\leq & C h\left\|z_{h}\right\|_{0, \Omega}\left\|\nabla z_{h}\right\|_{0, h} \\
\leq & \frac{1}{8}\left\|z_{h}\right\|_{0, \Omega}^{2}+C h^{2}\left\|\nabla z_{h}\right\|_{0, h}^{2} .
\end{aligned}
$$

Now we consider the $\mathcal{A}_{h}^{p}$-approximation. Fix $y_{h} \in \mathcal{A}_{h}^{p}$. We have as above that $T_{\mathcal{F}}^{p}\left(z_{h}\right)=0$ for any element face $\mathcal{F} \subset \partial \Omega$, so (cf. (4.8))

$$
\begin{aligned}
I_{1} & \equiv \sum_{\mathcal{R} \in \tau_{h}} \int_{\partial \mathcal{R}}\left|z_{h}(x)-T_{\mathcal{F}}^{p}\left(z_{h}\right)\right|^{2}(w \cdot x)(w \cdot \nu) d S \\
& \quad+2 \sum_{\mathcal{R} \in \tau_{h}} \sum_{\mathcal{F} \subset \partial \mathcal{R}} \int_{\mathcal{F}} T_{\mathcal{F}}^{p}\left(z_{h}\right) \cdot\left[z_{h}(x)-T_{\mathcal{F}}^{p}\left(z_{h}\right)\right](w \cdot x)\left(\left.w \cdot \nu\right|_{\mathcal{F}}\right) d S \\
& \equiv J_{1}^{p}+2 J_{2}^{p} .
\end{aligned}
$$


It similarly follows from (3.12) that

$$
\begin{aligned}
\left|J_{1}^{p}\right| & \equiv\left|\sum_{\mathcal{R} \in \tau_{h}} \sum_{\mathcal{F} \subset \partial \mathcal{R}} \int_{\mathcal{F}}\right| z_{h}(x)-\left.T_{\mathcal{F}}^{p}\left(z_{h}\right)\right|^{2}(w \cdot x)\left(\left.w \cdot \nu\right|_{\mathcal{F}}\right) d S \mid \\
& \leq C h\left\|\nabla z_{h}\right\|_{0, h}^{2} .
\end{aligned}
$$

Let us fix again $\mathcal{R}=\left[\alpha_{1}-l_{1}, \alpha_{1}+l_{1}\right] \times\left[\alpha_{2}-l_{2}, \alpha_{2}+l_{2}\right] \times\left[\alpha_{3}-l_{3}, \alpha_{3}+l_{3}\right] \in \tau_{h}$ and a pair of its faces $\mathcal{F}_{ \pm}=\left[\alpha_{1}-l_{1}, \alpha_{1}+l_{1}\right] \times\left[\alpha_{2}-l_{2}, \alpha_{2}+l_{2}\right] \times\left\{\alpha_{3} \pm l_{3}\right\}$. We have that $g_{\nu}(x)= \pm g(x)$ on $\mathcal{F}_{ \pm}$, where $g(x)=\left(w \cdot e_{3}\right)(w \cdot x)$. We also have by $(3.6)$ that

$$
z_{h}\left(x_{1}, x_{2}, \alpha_{3}+l_{3}\right)-z_{h}\left(\alpha_{1}, \alpha_{2}, \alpha_{3}+l_{3}\right)=z_{h}\left(x_{1}, x_{2}, \alpha_{3}-l_{3}\right)-z_{h}\left(\alpha_{1}, \alpha_{2}, \alpha_{3}-l_{3}\right) .
$$

It then follows from the above identity, the inverse inequality (3.9), and (3.12) that

$$
\begin{aligned}
\mid \int_{\mathcal{F}_{+}} & z_{h}\left(c_{\mathcal{F}_{+}}\right) \cdot\left[z_{h}(x)-z_{h}\left(c_{\mathcal{F}_{+}}\right)\right] g(x) d S \\
& \quad-\int_{\mathcal{F}_{-}} z_{h}\left(c_{\mathcal{F}_{-}}\right) \cdot\left[z_{h}(x)-z_{h}\left(c_{\mathcal{F}_{-}}\right)\right] g(x) d S \mid \\
= & \mid \int_{\alpha_{1}-l_{1}}^{\alpha_{1}+l_{1}} \int_{\alpha_{2}-l_{2}}^{\alpha+l_{2}}\left[z_{h}\left(x_{1}, x_{2}, \alpha_{3}+l_{3}\right)-z_{h}\left(\alpha_{1}, \alpha_{2}, \alpha_{3}+l_{3}\right)\right] \\
& \cdot\left[g\left(x_{1}, x_{2}, \alpha_{3}+l_{3}\right) z_{h}\left(\alpha_{1}, \alpha_{2}, \alpha_{3}+l_{3}\right)\right. \\
& \left.\quad-g\left(x_{1}, x_{2}, \alpha_{3}-l_{3}\right) z_{h}\left(\alpha_{1}, \alpha_{2}, \alpha_{3}-l_{3}\right)\right] d x_{1} d x_{2} \mid \\
= & \mid \int_{\alpha_{1}-l_{1}}^{\alpha_{1}+l_{1}} \int_{\alpha_{2}-l_{2}}^{\alpha_{2}+l_{2}}\left\{\left[z_{h}\left(x_{1}, x_{2}, \alpha_{3}+l_{3}\right)-z_{h}\left(\alpha_{1}, \alpha_{2}, \alpha_{3}+l_{3}\right)\right]\right. \\
& \left.\cdot\left[\int_{\alpha_{3}-l_{3}}^{\alpha_{3}+l_{3}} \frac{\partial}{\partial x_{3}}\left(g(x) z_{h}\left(\alpha_{1}, \alpha_{2}, x_{3}\right)\right) d x_{3}\right]\right\} d x_{1} d x_{2} \mid \\
\leq & C h\left\|z_{h}\right\|_{1, \infty, \mathcal{R}} \int_{\mathcal{F}_{+}}\left|z_{h}(x)-z_{h}\left(c_{\mathcal{F}_{+}}\right)\right| d S \\
\leq & C h^{2}\left\|z_{h}\right\|_{1, \infty, \mathcal{R}}\left(\int_{\mathcal{F}_{+}}\left|z_{h}(x)-z_{h}\left(c_{\mathcal{F}_{+}}\right)\right|^{2} d S\right)^{\frac{1}{2}} \\
\leq & C h\left(\left\|z_{h}\right\|_{0, \mathcal{R}}\left\|\nabla z_{h}\right\|_{0, \mathcal{R}}+\left\|\nabla z_{h}\right\|_{0, \mathcal{R}}^{2}\right) .
\end{aligned}
$$

This argument also applies to other pairs of faces of $\mathcal{R} \in \tau_{h}$. Hence, we can also conclude in this case that

$$
\begin{aligned}
\left|J_{2}^{p}\right| & \leq C h\left\|z_{h}\right\|_{0, \Omega}\left\|\nabla z_{h}\right\|_{0, h}+C h\left\|\nabla z_{h}\right\|_{0, h}^{2} \\
& \leq \frac{1}{8} \int_{\Omega}\left|z_{h}(x)\right|^{2} d x+C h\left\|\nabla z_{h}\right\|_{0, h}^{2} .
\end{aligned}
$$

The assertion of the theorem now follows from (4.6)-(4.15).

A local trace inequality was used in [21] to derive estimates for a nonconforming finite element approximation of a variational problem. But even an improved version of such a local result (cf. Lemma 3.4 in [24]) cannot be applied here to our 
situation. We thus give a global version of a discrete trace theorem for our finite element deformations.

Theorem 4.3. There exists a constant $C>0$ such that for any rectangular parallelepiped $\omega \subset \bar{\Omega}$ which is a union of elements $\mathcal{R}$ of a rectangular mesh $\tau_{h}$,

$$
\begin{aligned}
\sum_{\mathcal{R} \subset \omega, \partial \mathcal{R} \cap \partial \omega \neq \emptyset} \sum_{\mathcal{F} \subset \partial \mathcal{R} \cap \partial \omega} h^{2}\left|y_{h}\left(c_{\mathcal{F}}\right)-F_{\lambda} c_{\mathcal{F}}\right|^{2} \leq \frac{C}{\Lambda(\omega)} \int_{\omega}\left|y_{h}(x)-F_{\lambda} x\right|^{2} d x \\
+C\left(\int_{\omega}\left|y_{h}(x)-F_{\lambda} x\right|^{2} d x\right)^{\frac{1}{2}}\left(\sum_{\mathcal{R} \subset \omega} \int_{\mathcal{R}}\left\|\nabla y_{h}(x)-F_{\lambda}\right\|^{2} d x\right)^{\frac{1}{2}}
\end{aligned}
$$

for all $y_{h} \in \mathcal{A}_{h}^{p}$, and

$$
\begin{aligned}
\sum_{\mathcal{R} \subset \omega, \partial \mathcal{R} \cap \partial \omega \neq \emptyset} & \sum_{\mathcal{F} \subset \partial \mathcal{R} \cap \partial \omega} \int_{\mathcal{F}}\left|y_{h}(x)-F_{\lambda} x\right|^{2} d S \\
\leq & \frac{C}{\Lambda(\omega)} \int_{\omega}\left|y_{h}(x)-F_{\lambda} x\right|^{2} d x+C h \sum_{\mathcal{R} \subset \omega} \int_{\mathcal{R}}\left\|\nabla y_{h}(x)-F_{\lambda}\right\|^{2} d x \\
+ & C\left(\int_{\omega}\left|y_{h}(x)-F_{\lambda} x\right|^{2} d x\right)^{\frac{1}{2}}\left(\sum_{\mathcal{R} \subset \omega} \int_{\mathcal{R}}\left\|\nabla y_{h}(x)-F_{\lambda}\right\|^{2} d x\right)^{\frac{1}{2}}
\end{aligned}
$$

for all $y_{h} \in \mathcal{A}_{h}^{a}$, where $\Lambda(\omega)$ is the length of the shortest edge of $\omega$.

Proof. Assume that $\omega=\left[\omega_{1}^{-}, \omega_{1}^{+}\right] \times\left[\omega_{2}^{-}, \omega_{2}^{+}\right] \times\left[\omega_{3}^{-}, \omega_{3}^{+}\right]$. Fix $y_{h} \in \mathcal{A}_{h}$ and set $z_{h}(x)=y_{h}(x)-F_{\lambda} x, x \in \Omega$. Also, fix an arbitrary element face $\mathcal{F}_{0} \subset \partial \omega$ of an element $\mathcal{R} \subset \omega$. Assume without loss of generality that the corresponding unit exterior normal at $\mathcal{F}_{0}$ with respect to $\partial \omega$ is $\nu=\left.\nu\right|_{\mathcal{F}_{0}}=-e_{1}$. Denote by

$$
\mathcal{S}_{0}=\left\{x+y: x \in \mathcal{F}_{0} \text { and } y=s e_{1} \text { where } s \in\left[0, \omega_{1}^{+}-\omega_{1}^{-}\right]\right\} \subset \omega
$$

the cylinder composed of elements of $\tau_{h}$ with generating line parallel to $e_{1}$, one base $\mathcal{F}_{0} \subset \omega$, and the other base also on $\partial \omega$. We denote the corresponding height (the length of the generating line segment) of the cylinder $\mathcal{S}_{0}$ by $\Lambda_{1}=\omega_{1}^{+}-\omega_{1}^{-}$. Notice that $\Lambda_{1}$ is in fact the length of one edge of the rectangular parallelepiped $\omega$. Suppose further that the element faces which are in the cylinder $\mathcal{S}_{0}$ and are parallel to $\mathcal{F}_{0}$ are given by $\mathcal{F}_{i}, i=0, \cdots, k$, and that these faces lie respectively in the planes $x_{1}=\alpha_{1}^{(i)}$, for some $\omega_{1}^{-}=\alpha_{1}^{(0)}<\cdots<\alpha_{1}^{(k)}=\omega_{1}^{+}$.

Case 1. $y_{h} \in \mathcal{A}_{h}^{p}$. Denoting by $c_{\mathcal{F}_{i}}$ the center of the face $\mathcal{F}_{i}$ for $i=0, \cdots, k$, we have by the fact $\Lambda_{1}=\alpha_{1}^{(k)}-\alpha_{1}^{(0)}$ that

$$
\begin{aligned}
\sum_{i=0}^{k-1} & {\left[\left(\alpha_{1}^{(k)}-\alpha_{1}^{(i+1)}\right)\left|z_{h}\left(c_{\mathcal{F}_{i+1}}\right)\right|^{2}-\left(\alpha_{1}^{(k)}-\alpha_{1}^{(i)}\right)\left|z_{h}\left(c_{\mathcal{F}_{i}}\right)\right|^{2}\right] } \\
& =-\Lambda_{1}\left|z_{h}\left(c_{\mathcal{F}_{0}}\right)\right|^{2} .
\end{aligned}
$$

If $0 \leq i \leq k-1$, then

$$
\begin{aligned}
& \left.\left|\left(\alpha_{1}^{(k)}-\alpha_{1}^{(i+1)}\right)\right| z_{h}\left(c_{\mathcal{F}_{i+1}}\right)\right|^{2}-\left(\alpha_{1}^{(k)}-\alpha_{1}^{(i)}\right)\left|z_{h}\left(c_{\mathcal{F}_{i}}\right)\right|^{2} \mid \\
& \quad=\left.\left|\left(\alpha_{1}^{(k)}-\alpha_{1}^{(i)}\right)\left[\left|z_{h}\left(c_{\mathcal{F}_{i+1}}\right)\right|^{2}-\left|z_{h}\left(c_{\mathcal{F}_{i}}\right)\right|^{2}\right]+\left(\alpha_{1}^{(i)}-\alpha_{1}^{(i+1)}\right)\right| z_{h}\left(c_{\mathcal{F}_{i+1}}\right)\right|^{2} \mid \\
& \quad \leq \Lambda_{1}\left|\left[z_{h}\left(c_{\mathcal{F}_{i+1}}\right)-z_{h}\left(c_{\mathcal{F}_{i}}\right)\right] \cdot\left[z_{h}\left(c_{\mathcal{F}_{i+1}}\right)+z_{h}\left(c_{\mathcal{F}_{i}}\right)\right]\right|+h\left|z_{h}\left(c_{\mathcal{F}_{i+1}}\right)\right|^{2} .
\end{aligned}
$$


This, together with (4.18) and the inverse inequality (3.9), leads to

$$
\begin{aligned}
h^{2}\left|z_{h}\left(c_{\mathcal{F}_{0}}\right)\right|^{2} & \leq h^{3} \sum_{\mathcal{R} \subset \mathcal{S}_{0}}\left[\left\|\nabla z_{h}\right\|_{0, \infty, \mathcal{R}}\left\|z_{h}\right\|_{0, \infty, \mathcal{R}}+\frac{1}{\Lambda_{1}}\left\|z_{h}\right\|_{0, \infty, \mathcal{R}}^{2}\right] \\
& \leq C \sum_{\mathcal{R} \subset \mathcal{S}_{0}}\left[\left\|\nabla z_{h}\right\|_{0, \mathcal{R}}\left\|z_{h}\right\|_{0, \mathcal{R}}+\frac{1}{\Lambda(\omega)}\left\|z_{h}\right\|_{0, \mathcal{R}}^{2}\right] .
\end{aligned}
$$

Case 2. $y_{h} \in \mathcal{A}_{h}^{a}$. Noting that $\alpha_{1}^{(k)}-\alpha_{1}^{(0)}=\Lambda_{1}$, we have

$$
\begin{aligned}
\sum_{\mathcal{R} \subset \mathcal{S}_{0}} & \int_{\mathcal{R}} \frac{\partial}{\partial x_{1}}\left[\left(\alpha_{1}^{(k)}-x_{1}\right)\left|z_{h}(x)\right|^{2}\right] d x \\
& =\sum_{\mathcal{R} \subset \mathcal{S}_{0}} \int_{\partial \mathcal{R}}\left(\alpha_{1}^{(k)}-x_{1}\right)\left|z_{h}(x)\right|^{2}\left(\nu \cdot e_{1}\right) d S \\
& =-\Lambda_{1} \int_{\mathcal{F}_{0}}\left|z_{h}(x)\right|^{2} d S+\sum_{i=1}^{k-1}\left(\alpha_{1}^{(k)}-\alpha_{1}^{(i)}\right) \int_{\mathcal{F}_{i}}\left[\left|z_{h}^{+}(x)\right|^{2}-\left|z_{h}^{-}(x)\right|^{2}\right] d S,
\end{aligned}
$$

where for a fixed face $\mathcal{F}_{i}, 1 \leq i \leq k-1$, we denote by $z_{h}^{ \pm}$the restriction of $z_{h}$ to $\mathcal{F}_{i}$ for $z_{h}$ defined on the adjacent element sharing the common face $\mathcal{F}_{i}$ such that the corresponding unit exterior normal of the element boundary $\nu$ satisfies $\left.\nu\right|_{\mathcal{F}_{i}}= \pm e_{1}$. Consequently, we have that

$$
\begin{aligned}
\int_{\mathcal{F}_{0}}\left|z_{h}(x)\right|^{2} d S & \\
\leq & \sum_{\mathcal{R} \subset \mathcal{S}_{0}} \int_{\mathcal{R}}\left[\frac{1}{\Lambda_{1}}\left|z_{h}(x)\right|^{2}+2\left|z_{h}(x)\right|\left\|\nabla z_{h}(x)\right\|\right] d x \\
& \quad+\sum_{i=1}^{k-1}\left|\int_{\mathcal{F}_{i}}\left[\left|z_{h}^{+}(x)\right|^{2}-\left|z_{h}^{-}(x)\right|^{2}\right] d S\right| \\
= & \sum_{\mathcal{R} \subset \mathcal{S}_{0}}\left(\frac{1}{\Lambda_{1}}\left\|z_{h}\right\|_{0, \mathcal{R}}^{2}+2\left\|z_{h}\right\|_{0, \mathcal{R}}\left\|\nabla z_{h}\right\|_{0, \mathcal{R}}\right) \\
& +\sum_{i=1}^{k-1} \mid \int_{\mathcal{F}_{i}}\left[\left|z_{h}^{+}(x)-T_{\mathcal{F}_{i}}^{a}\left(z_{h}\right)+T_{\mathcal{F}_{i}}^{a}\left(z_{h}\right)\right|^{2}\right. \\
\quad & \left.\quad\left|z_{h}^{-}(x)-T_{\mathcal{F}_{i}}^{a}\left(z_{h}\right)+T_{\mathcal{F}_{i}}^{a}\left(z_{h}\right)\right|^{2}\right] d S \mid \\
= & \sum_{\mathcal{R} \subset \mathcal{S}_{0}}\left(\frac{1}{\Lambda_{1}}\left\|z_{h}\right\|_{0, \mathcal{R}}^{2}+2\left\|z_{h}\right\|_{0, \mathcal{R}}\left\|\nabla z_{h}\right\|_{0, \mathcal{R}}^{2}\right) \\
& +\sum_{i=1}^{k-1}\left|\int_{\mathcal{F}_{i}}\left[\left|z_{h}^{+}(x)-T_{\mathcal{F}_{i}}^{a}\left(z_{h}\right)\right|^{2}-\left|z_{h}^{-}(x)-T_{\mathcal{F}_{i}}^{a}\left(z_{h}\right)\right|^{2}\right] d S\right| \\
\leq & C \sum_{\mathcal{R} \subset \mathcal{S}_{0}}\left[\frac{1}{\Lambda(\omega)}\left\|z_{h}\right\|_{0, \mathcal{R}}^{2}+\left\|z_{h}\right\|_{0, \mathcal{R}}\left\|\nabla z_{h}\right\|_{0, \mathcal{R}}+h\left\|\nabla z_{h}\right\|_{0, \mathcal{R}}^{2}\right]
\end{aligned}
$$

where in the last step we used (3.12).

Since every such cylinder $\mathcal{S}_{0} \subset \omega$ will only be used twice corresponding to its two bases on $\partial \omega$, we therefore obtain (4.16) and (4.17) from (4.19) and (4.20), 
respectively, by summing over all boundary faces $\mathcal{F}_{0} \subset \partial \omega$ of elements $\mathcal{R} \subset \omega$ such that $\partial \mathcal{R} \cap \partial \omega \neq \emptyset$.

Remark 4.4. We can generalize the above theorem to cover more general closed subdomains $\omega \subset \bar{\Omega}$ which are still unions of rectangular elements of $\tau_{h}$. For such an $\omega$ we denote by $\Lambda(\omega)$ the smallest height of all cylinders $\mathcal{S}_{0} \subset \omega$ composed of elements of $\tau_{h}$ which have generating lines parallel to the coordinate axes and for which both bases lie in the boundary $\partial \omega$. Both of the inequalities (4.16) and (4.17) remain valid.

\section{Approximation of Limiting MaCroscopic Deformations}

We define

$$
\mathcal{E}_{h}\left(y_{h}\right)=\sum_{\mathcal{R} \in \tau_{h}} \int_{\mathcal{R}} \phi\left(\nabla y_{h}(x)\right) d x, \quad \forall y_{h} \in \mathcal{A}_{h}
$$

The following result which will be frequently used is a direct consequence of the quadratic growth rate of the energy density around the energy wells (2.2).

Lemma 5.1. We have

$$
\sum_{\mathcal{R} \in \tau_{h}} \int_{\mathcal{R}}\left\|\nabla y_{h}(x)-\pi\left(\nabla y_{h}(x)\right)\right\|^{2} d x \leq \kappa^{-1} \mathcal{E}_{h}\left(y_{h}\right), \quad \forall y_{h} \in \mathcal{A}_{h} .
$$

In the following lemma, we recall that we have assumed that

$$
F_{1}=F_{0}+a \otimes n,
$$

and that we have assumed without loss of generality in the cubic to tetragonal case by Lemma 2.1 that

$$
n=\frac{1}{\sqrt{2}}\left(e_{1}+e_{2}\right) .
$$

Lemma 5.2. For any $w \in \mathbb{R}^{3}$ satisfying $w \cdot n=0$, there exists a constant $C>0$ such that

$$
\sum_{\mathcal{R} \in \tau_{h}} \int_{\mathcal{R}}\left|\left[\pi\left(\nabla y_{h}(x)\right)-F_{\lambda}\right] w\right|^{2} d x \leq C \mathcal{E}_{h}\left(y_{h}\right)^{\frac{1}{2}}, \quad \forall y_{h} \in \mathcal{A}_{h} .
$$

Proof. We first consider the orthorhombic to monoclinic transformation. In this case we have

$$
\pi(F) \in \mathrm{SO}(3) F_{0} \cup \mathrm{SO}(3) F_{1}, \quad \forall F \in \mathbb{R}^{3 \times 3} .
$$

Consequently, we have by the rank-one connection (5.1) and by the identity

$$
F_{\lambda}=(1-\lambda) F_{0}+\lambda F_{1}=F_{0}+\lambda a \otimes n
$$

that

$$
|\pi(F) w|=\left|F_{0} w\right|=\left|F_{1} w\right|=\left|F_{\lambda} w\right|, \quad \forall F \in \mathbb{R}^{3 \times 3},
$$


for any $w \in \mathbb{R}^{3}$ such that $w \cdot n=0$. It then follows from Theorem 4.1, the CauchySchwarz inequality, the identity (5.4), and (2.2) that for any $y_{h} \in \mathcal{A}_{h}$,

$$
\begin{aligned}
\sum_{\mathcal{R} \in \tau_{h}} \int_{\mathcal{R}}\left|\left[\pi\left(\nabla y_{h}(x)\right)-F_{\lambda}\right] w\right|^{2} d x \\
=2 F_{\lambda} w \cdot \sum_{\mathcal{R} \in \tau_{h}} \int_{\mathcal{R}}\left[F_{\lambda}-\pi\left(\nabla y_{h}(x)\right)\right] w d x \\
=2 F_{\lambda} w \cdot \sum_{\mathcal{R} \in \tau_{h}} \int_{\mathcal{R}}\left[\nabla y_{h}(x)-\pi\left(\nabla y_{h}(x)\right)\right] w d x \\
\leq 2\left|F_{\lambda} w\right|(\operatorname{meas} \Omega)^{1 / 2}\left[\sum_{\mathcal{R} \in \tau_{h}} \int_{\mathcal{R}}\left\|\nabla y_{h}(x)-\pi\left(\nabla y_{h}(x)\right)\right\|^{2} d x\right]^{\frac{1}{2}} \\
\leq 2\left|F_{0} w\right|(\operatorname{meas} \Omega)^{1 / 2} \kappa^{-1 / 2} \mathcal{E}_{h}\left(y_{h}\right)^{\frac{1}{2}},
\end{aligned}
$$

which implies (5.3) for the orthorhombic to monoclinic transformation.

Now we consider the cubic to tetragonal transformation. Set

$$
w_{1}=e_{1}-e_{2}+e_{3} \quad \text { and } \quad w_{2}=e_{1}-e_{2}-e_{3} .
$$

It is easy to check that

$$
w_{1} \cdot n=w_{2} \cdot n=0
$$

and

$$
\left|U_{i} w_{j}\right|=\sqrt{2 \eta_{1}^{2}+\eta_{2}^{2}}, \quad i=1,2,3, j=1,2 .
$$

Consequently, we can obtain (5.4) and hence (5.5) again for $w=w_{1}$ and $w=w_{2}$, respectively. Thus, (5.3) is also proved for the cubic to tetragonal transformation since $\left\{w_{1}, w_{2}\right\}$ is an orthonormal basis for the two-dimensional subspace $\left\{w \in \mathbb{R}^{3}\right.$ : $w \cdot n=0\}$.

The following theorem is a direct consequence of the above two lemmas. It gives error bounds for the approximation of directional derivatives of deformations to the limiting macroscopic deformation gradient $F_{\lambda}$ in the direction tangential to the parallel layers of the laminate. It will play a key role in establishing all of the other error bounds.

Theorem 5.3. For any $w \in \mathbb{R}^{3}$ satisfying $w \cdot n=0$, there exists a constant $C>0$ such that

$$
\sum_{\mathcal{R} \in \tau_{h}} \int_{\mathcal{R}}\left|\left[\nabla y_{h}(x)-F_{\lambda}\right] w\right|^{2} d x \leq C\left[\mathcal{E}_{h}\left(y_{h}\right)^{\frac{1}{2}}+\mathcal{E}_{h}\left(y_{h}\right)\right], \quad \forall y_{h} \in \mathcal{A}_{h} .
$$

We now give error bounds for the strong $L^{2}$-approximation of deformations to the limiting macroscopic homogeneous deformation $F_{\lambda} x, x \in \Omega$.

Theorem 5.4. There is a constant $C>0$ such that

$$
\int_{\Omega}\left|y_{h}(x)-F_{\lambda} x\right|^{2} d x \leq C\left[\mathcal{E}_{h}\left(y_{h}\right)^{\frac{1}{2}}+\mathcal{E}_{h}\left(y_{h}\right)+h\right], \quad \forall y_{h} \in \mathcal{A}_{h} .
$$


Proof. For any $y_{h} \in \mathcal{A}_{h}$, we have by Lemma 5.1 that

$$
\begin{aligned}
\sum_{\mathcal{R} \in \tau_{h}} & \int_{\mathcal{R}}\left\|\nabla y_{h}(x)-F_{\lambda}\right\|^{2} d x \\
(5.6) \quad & \leq 2 \sum_{\mathcal{R} \in \tau_{h}} \int_{\mathcal{R}}\left\|\nabla y_{h}(x)-\pi\left(\nabla y_{h}(x)\right)\right\|^{2} d x+2 \sum_{\mathcal{R} \in \tau_{h}} \int_{\mathcal{R}}\left\|\pi\left(\nabla y_{h}(x)\right)-F_{\lambda}\right\|^{2} d x \\
& \leq C \mathcal{E}_{h}\left(y_{h}\right)+C,
\end{aligned}
$$

which together with Theorem 4.2 implies the desired inequality.

We now establish error bounds for the weak approximation of deformation gradients to the limiting macroscopic deformation gradient $F_{\lambda}$.

Theorem 5.5. For any rectangular parallelepiped $\omega \subset \bar{\Omega}$ whose boundary $\partial \omega$ is composed of faces parallel to the coordinate planes, there exists a constant $C=$ $C(\omega)>0$ such that for all $y_{h} \in \mathcal{A}_{h}$

$$
\left\|\sum_{\mathcal{R} \in \tau_{h}} \int_{\omega \cap \mathcal{R}}\left[\nabla y_{h}(x)-F_{\lambda}\right] d x\right\| \leq C\left[\mathcal{E}_{h}\left(y_{h}\right)^{\frac{1}{8}}+\mathcal{E}_{h}\left(y_{h}\right)^{\frac{1}{2}}+h^{\frac{1}{4}}\right] .
$$

Proof. Denoting

$$
\omega_{h}=\bigcup\left\{\mathcal{R} \in \tau_{h}: \mathcal{R} \subset \omega\right\},
$$

we have for any $y_{h} \in \mathcal{A}_{h}$ that

$$
\begin{aligned}
& \sum_{\mathcal{R} \in \tau_{h}} \int_{\omega \cap \mathcal{R}}\left[\nabla y_{h}(x)-F_{\lambda}\right] d x \\
& \quad=\sum_{\mathcal{R} \in \tau_{h}, \mathcal{R} \subset \omega_{h}} \int_{\mathcal{R}}\left[\nabla y_{h}(x)-F_{\lambda}\right] d x+\sum_{\mathcal{R} \in \tau_{h}} \int_{\left(\omega-\omega_{h}\right) \cap \mathcal{R}}\left[\nabla y_{h}(x)-F_{\lambda}\right] d x \\
& \quad \equiv K_{1}+K_{2} .
\end{aligned}
$$

Since

$$
\text { meas }\left(\omega-\omega_{h}\right) \leq C h,
$$

we can estimate $K_{2}$ by virtue of the triangle inequality, the Cauchy-Schwarz inequality, and Lemma 5.1 to get

$$
\begin{aligned}
\left\|K_{2}\right\| \equiv & \left\|\sum_{\mathcal{R} \in \tau_{h}} \int_{\left(\omega-\omega_{h}\right) \cap \mathcal{R}}\left[\nabla y_{h}(x)-F_{\lambda}\right] d x\right\| \\
\leq & \left\|\sum_{\mathcal{R} \in \tau_{h}} \int_{\left(\omega-\omega_{h}\right) \cap \mathcal{R}}\left[\nabla y_{h}(x)-\pi\left(\nabla y_{h}(x)\right)\right] d x\right\| \\
& +\left\|\sum_{\mathcal{R} \in \tau_{h}} \int_{\left(\omega-\omega_{h}\right) \cap \mathcal{R}}\left[\pi\left(\nabla y_{h}(x)\right)-F_{\lambda}\right] d x\right\| \\
\leq & C h^{\frac{1}{2}}\left[\sum_{\mathcal{R} \in \tau_{h}} \int_{\mathcal{R}}\left\|\nabla y_{h}(x)-\pi\left(\nabla y_{h}(x)\right)\right\|^{2} d x\right]^{\frac{1}{2}}+C h \\
\leq & C h^{\frac{1}{2}} \mathcal{E}_{h}\left(y_{h}\right)^{\frac{1}{2}}+C h .
\end{aligned}
$$


To estimate $K_{1}$ we first assume that $y_{h} \in \mathcal{A}_{h}^{a}$. It then follows from the divergence theorem and the definition of the $\mathcal{A}_{h}^{a}$-approximation that

$$
\begin{aligned}
K_{1} & \equiv \sum_{\mathcal{R} \in \tau_{h}, \mathcal{R} \subset \omega_{h}} \int_{\mathcal{R}}\left[\nabla y_{h}(x)-F_{\lambda}\right] d x \\
& =\sum_{\mathcal{R} \in \tau_{h}, \mathcal{R} \subset \omega_{h}} \int_{\partial \mathcal{R}}\left[y_{h}(x)-F_{\lambda} x\right] \otimes \nu d S \\
& =\sum_{\mathcal{R} \subset \omega_{h}, \partial \mathcal{R} \cap \partial \omega_{h} \neq \emptyset} \sum_{\mathcal{F} \subset \partial \mathcal{R} \cap \partial \omega_{h}} \int_{\mathcal{F}}\left[y_{h}(x)-F_{\lambda} x\right] \otimes \nu d S .
\end{aligned}
$$

Since $\omega_{h} \subset \bar{\Omega}$ is a rectangular parallelepiped which is a union of elements in $\tau_{h}$, we have by the Cauchy-Schwarz inequality and (4.17) that

$$
\begin{gathered}
\left\|K_{1}\right\| \leq \sum_{\mathcal{R} \subset \omega_{h}, \partial \mathcal{R} \cap \partial \omega_{h} \neq \emptyset} \sum_{\mathcal{F} \subset \partial \mathcal{R} \cap \partial \omega_{h}} \int_{\mathcal{F}}\left|y_{h}(x)-F_{\lambda} x\right| d S \\
\leq\left(\operatorname{meas} \partial \omega_{h}\right)^{\frac{1}{2}}\left[\sum_{\mathcal{R} \subset \omega_{h}, \partial \mathcal{R} \cap \partial \omega_{h} \neq \emptyset} \sum_{\mathcal{F} \subset \partial \mathcal{R} \cap \partial \omega_{h}} \int_{\mathcal{F}}\left|y_{h}(x)-F_{\lambda} x\right|^{2} d S\right]^{\frac{1}{2}} \\
\leq C(\operatorname{meas} \partial \omega)^{\frac{1}{2}}\left\{\frac{1}{\Lambda\left(\omega_{h}\right)} \int_{\omega_{h}}\left|y_{h}(x)-F_{\lambda} x\right|^{2} d x+h \sum_{\mathcal{R} \subset \omega_{h}} \int_{\mathcal{R}}\left\|\nabla y_{h}(x)-F_{\lambda}\right\|^{2} d x\right. \\
\left.\quad+\left(\int_{\omega_{h}}\left|y_{h}(x)-F_{\lambda} x\right|^{2} d x\right)^{\frac{1}{2}}\left(\sum_{\mathcal{R} \subset \omega_{h}} \int_{\mathcal{R}}\left\|\nabla y_{h}(x)-F_{\lambda}\right\|^{2} d x\right)^{\frac{1}{2}}\right\}^{\frac{1}{2}} \\
\leq C\left\{\frac{1}{\Lambda\left(\omega_{h}\right)} \int_{\Omega}\left|y_{h}(x)-F_{\lambda} x\right|^{2} d x+h \sum_{\mathcal{R} \in \tau_{h}} \int_{\mathcal{R}}\left\|\nabla y_{h}(x)-F_{\lambda}\right\|^{2} d x\right. \\
\left.\quad+\left(\int_{\Omega}\left|y_{h}(x)-F_{\lambda} x\right|^{2} d x\right)^{\frac{1}{2}}\left(\sum_{\mathcal{R} \in \tau_{h}} \int_{\mathcal{R}}\left\|\nabla y_{h}(x)-F_{\lambda}\right\|^{2} d x\right)^{\frac{1}{2}}\right\}^{\frac{1}{2}}
\end{gathered}
$$

since $\Lambda(\omega) \leq C \Lambda\left(\omega_{h}\right)$. This, together with (5.6) and Theorem (5.4), implies that

$$
\left\|K_{1}\right\| \leq C\left[\mathcal{E}_{h}\left(y_{h}\right)^{\frac{1}{8}}+\mathcal{E}_{h}\left(y_{h}\right)^{\frac{1}{2}}+h^{\frac{1}{4}}\right] .
$$

Now let us assume that $y_{h} \in \mathcal{A}_{h}^{p}$. By the same argument as in the proof of Theorem 4.1, cf. (4.3) and (4.4), we have

$$
\begin{aligned}
K_{1} \equiv & \sum_{\mathcal{R} \in \tau_{h}, \mathcal{R} \subset \omega_{h}} \int_{\mathcal{R}}\left[\nabla y_{h}(x)-F_{\lambda}\right] d x \\
= & \sum_{\mathcal{R} \in \tau_{h}, \mathcal{R} \subset \omega_{h}} \int_{\partial \mathcal{R}}\left[y_{h}(x)-F_{\lambda} x\right] \otimes \nu d S \\
= & \sum_{\mathcal{R} \in \tau_{h}, \mathcal{R} \subset \omega_{h}} \sum_{\mathcal{F} \subset \partial \mathcal{R}} \int_{\mathcal{F}}\left\{\left[y_{h}(x)-F_{\lambda} x\right]-\left[y_{h}\left(c_{\mathcal{F}}\right)-F_{\lambda} c_{\mathcal{F}}\right]\right\} \otimes \nu d S \\
& \quad+\sum_{\mathcal{R} \in \tau_{h}, \mathcal{R} \subset \omega_{h}} \sum_{\mathcal{F} \subset \partial \mathcal{R}} \int_{\mathcal{F}}\left[y_{h}\left(c_{\mathcal{F}}\right)-F_{\lambda} c_{\mathcal{F}}\right] \otimes \nu d S
\end{aligned}
$$




$$
\begin{aligned}
& =\sum_{\mathcal{R} \in \tau_{h}, \mathcal{R} \subset \omega_{h}} \sum_{\mathcal{F} \subset \partial \mathcal{R}} \int_{\mathcal{F}}\left[y_{h}\left(c_{\mathcal{F}}\right)-F_{\lambda} c_{\mathcal{F}}\right] \otimes \nu d S \\
& =\sum_{\mathcal{R} \subset \omega_{h}, \partial \mathcal{R} \cap \partial \omega_{h} \neq \emptyset} \sum_{\mathcal{F} \subset \partial \mathcal{R} \cap \partial \omega_{h}} \int_{\mathcal{F}}\left[y_{h}\left(c_{\mathcal{F}}\right)-F_{\lambda} c_{\mathcal{F}}\right] \otimes \nu d S .
\end{aligned}
$$

Using a similar argument to that for $y_{h} \in \mathcal{A}_{h}^{a}$, we can obtain (5.10) again for $y_{h} \in \mathcal{A}_{h}^{p}$ by (4.16), (5.6), and Theorem 5.4.

Finally, (5.7) follows from (5.8), (5.9), and (5.10).

\section{APPROXIMATION OF MARTENSITIC VARIANTS}

Let us now define the projection operator $\pi_{12}: \mathbb{R}^{3 \times 3} \rightarrow \mathcal{U}_{1} \cup \mathcal{U}_{2}$ by

$$
\left\|F-\pi_{12}(F)\right\|=\min _{G \in \mathcal{U}_{1} \cup \mathcal{U}_{2}}\|F-G\|, \quad \forall F \in \mathbb{R}^{3 \times 3}
$$

For the orthorhombic to monoclinic transformation, we note that $\pi_{12}=\pi$. The next lemma gives an estimate for $\pi_{12}-\pi$ for the cubic to tetragonal transformation by showing that the measure of the set of points in which the gradient of energy minimizing sequences of deformations is near $\mathcal{U}_{3}$ converges to zero. Thus, the next lemma reduces the three-well problem for the cubic to tetragonal transformation to a two-well problem.

Lemma 6.1. For the cubic to tetragonal transformation, there exists a constant $C>0$ such that

$$
\sum_{\mathcal{R} \in \tau_{h}} \int_{\mathcal{R}}\left\|\pi\left(\nabla y_{h}(x)\right)-\pi_{12}\left(\nabla y_{h}(x)\right)\right\|^{2} d x \leq C \mathcal{E}_{h}\left(y_{h}\right)^{\frac{1}{2}}, \quad \forall y \in \mathcal{A}_{h}
$$

Proof. We have by a simple calculation that

$$
\inf _{F \in \mathcal{U}_{3}}\left|\left[F-F_{\lambda}\right] e_{3}\right| \geq\left|\eta_{2}-\eta_{1}\right| .
$$

Denoting

$$
\Omega_{3}=\bigcup_{\mathcal{R} \in \tau_{h}}\left\{x \in \mathcal{R}: \pi\left(\nabla y_{h}(x)\right) \in \mathcal{U}_{3}\right\}
$$

for $y_{h} \in \mathcal{A}_{h}$, we have by Lemma 5.2 that

$$
\begin{aligned}
\operatorname{meas} \Omega_{3} & =\sum_{\mathcal{R} \in \tau_{h}} \operatorname{meas}\left\{x \in \mathcal{R}: \pi\left(\nabla y_{h}(x)\right) \in \mathcal{U}_{3}\right\} \\
& \leq\left|\eta_{2}-\eta_{1}\right|^{-2} \sum_{\mathcal{R} \in \tau_{h}} \int_{\mathcal{R}}\left|\left[\pi\left(\nabla y_{h}(x)\right)-F_{\lambda}\right] e_{3}\right|^{2} d x \\
& \leq C \mathcal{E}_{h}\left(y_{h}\right)^{\frac{1}{2}}
\end{aligned}
$$


since $e_{3} \cdot n=0$ (recall that $n=2^{-1 / 2}\left(e_{1}+e_{2}\right)$ ). The result (6.1) then follows from the inequality

$$
\begin{aligned}
& \sum_{\mathcal{R} \in \tau_{h}} \int_{\mathcal{R}}\left\|\pi(\nabla y(x))-\pi_{12}\left(\nabla y_{h}(x)\right)\right\|^{2} d x \\
& \quad=\sum_{\mathcal{R} \in \tau_{h}} \int_{\mathcal{R} \cap \Omega_{3}}\left\|\pi(\nabla y(x))-\pi_{12}\left(\nabla y_{h}(x)\right)\right\|^{2} d x \\
& \quad \leq 4\left(2 \eta_{1}^{2}+\eta_{2}^{2}\right) \text { meas } \Omega_{3} \\
& \quad \leq C \mathcal{E}_{h}\left(y_{h}\right)^{\frac{1}{2}}
\end{aligned}
$$

since $\|\pi(F)\|=\left\|\pi_{12}(F)\right\|=\sqrt{2 \eta_{1}^{2}+\eta_{2}^{2}}$ for all $F \in \mathbb{R}^{3 \times 3}$.

We next define the operators $\Theta: \mathbb{R}^{3 \times 3} \rightarrow \mathrm{SO}(3)$ and $\Pi: \mathbb{R}^{3 \times 3} \rightarrow\left\{F_{0}, F_{1}\right\}$ by the relation

$$
\pi_{12}(F)=\Theta(F) \Pi(F), \quad \forall F \in \mathbb{R}^{3 \times 3} .
$$

The following theorem gives an error bound for the convergence of deformation gradients to the set of variants $\left\{F_{0}, F_{1}\right\}$.

Theorem 6.2. There exists a constant $C>0$ such that

$$
\sum_{\mathcal{R} \in \tau_{h}} \int_{\mathcal{R}}\left\|\nabla y_{h}(x)-\Pi\left(\nabla y_{h}(x)\right)\right\|^{2} d x \leq C\left[\mathcal{E}_{h}\left(y_{h}\right)^{\frac{1}{2}}+\mathcal{E}_{h}\left(y_{h}\right)\right], \quad \forall y_{h} \in \mathcal{A}_{h} .
$$

Proof. For any $w \in \mathbb{R}^{3}$ such that $w \cdot n=0$, we have

$$
\Pi(F) w=F_{0} w=F_{1} w=F_{\lambda} w, \quad \forall F \in \mathbb{R}^{3 \times 3} .
$$

Thus, it follows from (6.3) that

$$
\begin{aligned}
& {[\Theta(F)-I] F_{0} w=[\Theta(F)-I] \Pi(F) w=\left[\pi_{12}(F)-F_{\lambda}\right] w} \\
& \quad=\left[\pi_{12}(F)-\pi(F)\right] w+\left[\pi(F)-F_{\lambda}\right] w, \quad \forall F \in \mathbb{R}^{3 \times 3} .
\end{aligned}
$$

We can then apply the triangle inequality to the above identity with $F=\nabla y_{h}(x)$, $x \in \mathcal{R}$, for any $y_{h} \in \mathcal{A}_{h}$ and any element $\mathcal{R} \in \tau_{h}$, and estimate the corresponding two terms by Lemma 6.1 and Lemma 5.2 to obtain for $w \cdot n=0$ that

$$
\begin{aligned}
\sum_{\mathcal{R} \in \tau_{h}} \int_{\mathcal{R}}\left|\left[\Theta\left(\nabla y_{h}(x)\right)-I\right] F_{0} w\right|^{2} d x \\
\leq 2 \sum_{\mathcal{R} \in \tau_{h}} \int_{\mathcal{R}}\left|\left[\pi_{12}\left(\nabla y_{h}(x)\right)-\pi\left(\nabla y_{h}(x)\right)\right] w\right|^{2} d x \\
\quad+2 \sum_{\mathcal{R} \in \tau_{h}} \int_{\mathcal{R}}\left|\left[\pi\left(\nabla y_{h}(x)\right)-F_{\lambda}\right] w\right|^{2} d x \\
\leq C \mathcal{E}_{h}\left(y_{h}\right)^{\frac{1}{2}}
\end{aligned}
$$

Choose $w_{1} \in \mathbb{R}^{3}$ and $w_{2} \in \mathbb{R}^{3}$ so that $w_{1} \cdot n=w_{2} \cdot n=0$ and $w_{1}, w_{2}$ are linearly independent. Set $m=F_{0} w_{1} \times F_{0} w_{2}$. Since

$$
Q m=Q F_{0} w_{1} \times Q F_{0} w_{2}, \quad \forall Q \in \mathrm{SO}(3),
$$

we have for all $F \in \mathbb{R}^{3 \times 3}$ that

$$
\begin{aligned}
& {[\Theta(F)-I] m=\left\{\Theta(F) F_{0} w_{1} \times \Theta(F) F_{0} w_{2}\right\}-\left\{F_{0} w_{1} \times F_{0} w_{2}\right\}} \\
& \quad=\left\{[\Theta(F)-I] F_{0} w_{1} \times \Theta(F) F_{0} w_{2}\right\}-\left\{F_{0} w_{1} \times[I-\Theta(F)] F_{0} w_{2}\right\} .
\end{aligned}
$$


This together with (6.4) implies that

$$
\sum_{\mathcal{R} \in \tau_{h}} \int_{\mathcal{R}}\left|\left[\Theta\left(\nabla y_{h}(x)\right)-I\right] m\right|^{2} d x \leq C \mathcal{E}_{h}\left(y_{h}\right)^{\frac{1}{2}}
$$

Now $\left\{F_{0} w_{1}, F_{0} w_{2}, m\right\}$ is a basis for $\mathbb{R}^{3}$, so we can conclude from (6.4) and (6.5) that for all $y_{h} \in \mathcal{A}_{h}$

$$
\sum_{\mathcal{R} \in \tau_{h}} \int_{\mathcal{R}}\left\|\left[\Theta\left(\nabla y_{h}(x)\right)-I\right]\right\|^{2} d x \leq C\left[\mathcal{E}_{h}\left(y_{h}\right)^{\frac{1}{2}}+\mathcal{E}_{h}\left(y_{h}\right)\right] .
$$

We complete the proof by applying the triangle inequality to the identity

$$
\begin{aligned}
F-\Pi(F) & =[F-\pi(F)]+\left[\pi(F)-\pi_{12}(F)\right]+\left[\pi_{12}(F)-\Pi(F)\right] \\
& =[F-\pi(F)]+\left[\pi(F)-\pi_{12}(F)\right]+[\Theta(F)-I] \Pi(F), \quad \forall F \in \mathbb{R}^{3 \times 3},
\end{aligned}
$$

with $F=\nabla y_{h}(x)$ for any $y_{h} \in \mathcal{A}_{h}, x \in \mathcal{R}$, and $\mathcal{R} \in \tau_{h}$, and by estimating the three terms by Lemma 5.1, Lemma 6.1, and (6.6).

\section{Approximation of Simply laminated microstructure}

For any subset $\omega \subset \Omega, \rho>0$, and $y_{h} \in \mathcal{A}_{h}$, we define the sets

$$
\begin{aligned}
& \omega_{\rho}^{0}\left(y_{h}\right)=\bigcup_{\mathcal{R} \in \tau_{h}}\left\{x \in \omega \cap \mathcal{R}: \Pi\left(\nabla y_{h}(x)\right)=F_{0} \text { and }\left\|\nabla y_{h}(x)-F_{0}\right\|<\rho\right\}, \\
& \omega_{\rho}^{1}\left(y_{h}\right)=\bigcup_{\mathcal{R} \in \tau_{h}}\left\{x \in \omega \cap \mathcal{R}: \Pi\left(\nabla y_{h}(x)\right)=F_{1} \text { and }\left\|\nabla y_{h}(x)-F_{1}\right\|<\rho\right\} .
\end{aligned}
$$

The following theorem states that for any rectangular parallelepiped $\omega \subset \bar{\Omega}$ and for any energy minimizing sequence $\left\{y_{h}\right\}$ the volume fraction that the piecewise defined gradient $\nabla y_{h}$ is near $F_{0}$ converges to $1-\lambda$ and the volume fraction that $\nabla y_{h}$ is near $F_{1}$ converges to $\lambda$.

Theorem 7.1. For any rectangular parallelepiped $\omega \subset \Omega$ whose faces are parallel to the coordinate planes, and any $\rho>0$, there exists a constant $C=C(\omega, \rho)>0$ such that for all $y_{h} \in \mathcal{A}_{h}$,

$$
\begin{aligned}
& \left|\frac{\operatorname{meas} \omega_{\rho}^{0}\left(y_{h}\right)}{\operatorname{meas} \omega}-(1-\lambda)\right|+\left|\frac{\operatorname{meas} \omega_{\rho}^{1}\left(y_{h}\right)}{\operatorname{meas} \omega}-\lambda\right| \\
& \leq C\left[\mathcal{E}_{h}\left(y_{h}\right)^{\frac{1}{8}}+\mathcal{E}_{h}\left(y_{h}\right)^{\frac{1}{2}}+h^{\frac{1}{4}}\right] .
\end{aligned}
$$

Proof. Fix $y_{h} \in \mathcal{A}_{h}$. It follows from the definition of $\omega_{\rho}^{0} \equiv \omega_{\rho}^{0}\left(y_{h}\right)$ and $\omega_{\rho}^{1} \equiv \omega_{\rho}^{1}\left(y_{h}\right)$ that

$$
\begin{aligned}
{[\text { meas }} & \left.\omega_{\rho}^{0}-(1-\lambda) \text { meas } \omega\right] F_{0}+\left[\text { meas } \omega_{\rho}^{1}-\lambda \text { meas } \omega\right] F_{1} \\
= & \sum_{\mathcal{R} \in \tau_{h}} \int_{\omega \cap \mathcal{R}}\left[\Pi\left(\nabla y_{h}(x)\right)-F_{\lambda}\right] d x \\
& -\sum_{\mathcal{R} \in \tau_{h}} \int_{\left(\omega-\left\{\omega_{\rho}^{0} \cup \omega_{\rho}^{1}\right\}\right) \cap \mathcal{R}} \Pi\left(\nabla y_{h}(x)\right) d x .
\end{aligned}
$$


We have by Theorem 6.1 and Theorem 5.5 that

$$
\begin{aligned}
& \left\|\sum_{\mathcal{R} \in \tau_{h}} \int_{\omega \cap \mathcal{R}}\left[\Pi\left(\nabla y_{h}(x)\right)-F_{\lambda}\right] d x\right\| \\
& \leq\left\|\sum_{\mathcal{R} \in \tau_{h}} \int_{\omega \cap \mathcal{R}}\left[\Pi\left(\nabla y_{h}(x)\right)-\nabla y_{h}(x)\right] d x\right\|+\left\|\sum_{\mathcal{R} \in \tau_{h}} \int_{\omega \cap \mathcal{R}}\left[\nabla y_{h}(x)-F_{\lambda}\right] d x\right\| \\
& \leq(\operatorname{meas} \omega)^{\frac{1}{2}}\left[\sum_{\mathcal{R} \in \tau_{h}} \int_{\mathcal{R}}\left\|\Pi\left(\nabla y_{h}(x)\right)-\nabla y_{h}(x)\right\|^{2} d x\right]^{\frac{1}{2}} \\
& \quad+\left\|\sum_{\mathcal{R} \in \tau_{h}} \int_{\omega \cap \mathcal{R}}\left[\nabla y_{h}(x)-F_{\lambda}\right] d x\right\| \\
& \leq C\left[\mathcal{E}_{h}\left(y_{h}\right)^{\frac{1}{8}}+\mathcal{E}_{h}\left(y_{h}\right)^{\frac{1}{2}}+h^{\frac{1}{4}}\right] .
\end{aligned}
$$

Since $\|\Pi(F)\|=\sqrt{2 \eta_{1}^{2}+\eta_{2}^{2}}$ for all $F \in \mathbb{R}^{3 \times 3}$, we can conclude by the definition of $\omega_{\rho}^{0}$ and $\omega_{\rho}^{1}$ and by Theorem 6.2 that

$$
\begin{aligned}
& \left\|\sum_{\mathcal{R} \in \tau_{h}} \int_{\left(\omega-\left\{\omega_{\rho}^{0} \cup \omega_{\rho}^{1}\right\}\right) \cap \mathcal{R}} \Pi\left(\nabla y_{h}(x)\right) d x\right\| \\
& \quad \leq C \operatorname{meas}\left(\omega-\left\{\omega_{\rho}^{0} \cup \omega_{\rho}^{1}\right\}\right) \\
& \leq \frac{C}{\rho} \sum_{\mathcal{R} \in \tau_{h}} \int_{\left(\omega-\left\{\omega_{\rho}^{0} \cup \omega_{\rho}^{1}\right\}\right) \cap \mathcal{R}}\left\|\Pi\left(\nabla y_{h}(x)\right)-\nabla y_{h}(x)\right\| d x \\
& \leq \frac{C(\operatorname{meas} \omega)^{\frac{1}{2}}}{\rho}\left[\sum_{\mathcal{R} \in \tau_{h}} \int_{\mathcal{R}}\left\|\Pi\left(\nabla y_{h}(x)\right)-\nabla y_{h}(x)\right\|^{2} d x\right]^{\frac{1}{2}} \\
& \leq C\left[\mathcal{E}_{h}\left(y_{h}\right)^{\frac{1}{4}}+\mathcal{E}_{h}\left(y_{h}\right)^{\frac{1}{2}}\right] .
\end{aligned}
$$

Therefore, we have by (7.3) and (7.4) that

$$
\begin{aligned}
& \|\left[\text { meas } \omega_{\rho}^{0}-(1-\lambda) \operatorname{meas} \omega\right] F_{0}+\left[\operatorname{meas} \omega_{\rho}^{1}-\lambda \operatorname{meas} \omega\right] F_{1} \| \\
& \leq C\left[\mathcal{E}_{h}\left(y_{h}\right)^{\frac{1}{8}}+\mathcal{E}_{h}\left(y_{h}\right)^{\frac{1}{2}}+h^{\frac{1}{4}}\right],
\end{aligned}
$$

which implies (7.1) because $F_{0}$ and $F_{1}$ are linearly independent.

We now denote by $\mathcal{V}$ the Sobolev space of all measurable functions $f(x, F)$ : $\Omega \times \mathbb{R}^{3 \times 3} \rightarrow \mathbb{R}$ such that

$$
\|f\|_{\mathcal{V}}^{2}=\int_{\Omega}\left[\operatorname{esssup}_{F \in \mathbb{R}^{3 \times 3}}\left\|\nabla_{F} f(x, F)\right\|\right]^{2} d x+\left\|G_{f}\right\|_{1, \Omega}^{2}<\infty
$$

where

$$
G_{f}(x)=f\left(x, F_{1}\right)-f\left(x, F_{0}\right), \quad x \in \Omega .
$$

The following theorem gives error bounds for the approximation of nonlinear integrals of deformation gradients which represent macroscopic thermodynamic densities. 
Theorem 7.2. There exists a constant $C>0$ such that

$$
\begin{aligned}
& \mid \sum_{\mathcal{R} \in \tau_{h}} \int_{\mathcal{R}}\left\{f\left(\left(x, \nabla y_{h}(x)\right)-\left[(1-\lambda) f\left(x, F_{0}\right)+\lambda f\left(x, F_{1}\right)\right]\right\} d x \mid\right. \\
& \quad \leq C\|f\|_{\mathcal{V}}\left[\mathcal{E}_{h}\left(y_{h}\right)^{\frac{1}{4}}+\mathcal{E}_{h}\left(y_{h}\right)^{\frac{1}{2}}+h^{\frac{1}{2}}\right], \quad \forall f \in \mathcal{V}, \forall y_{h} \in \mathcal{A}_{h} .
\end{aligned}
$$

Proof. We have

$$
\begin{aligned}
& \sum_{\mathcal{R} \in \tau_{h}} \int_{\mathcal{R}}\left\{f\left(\left(x, \nabla y_{h}(x)\right)-\left[(1-\lambda) f\left(x, F_{0}\right)+\lambda f\left(x, F_{1}\right)\right]\right\} d x\right. \\
& =\sum_{\mathcal{R} \in \tau_{h}} \int_{\mathcal{R}}\left[f\left(x, \nabla y_{h}(x)\right)-f\left(x, \Pi\left(\nabla y_{h}(x)\right)\right)\right] d x \\
& \quad+\sum_{\mathcal{R} \in \tau_{h}} \int_{\mathcal{R}}\left\{f\left(x, \Pi\left(\nabla y_{h}(x)\right)\right)-\left[(1-\lambda) f\left(x, F_{0}\right)+\lambda f\left(x, F_{1}\right)\right]\right\} d x \\
& =M_{1}+M_{2} .
\end{aligned}
$$

The first term $M_{1}$ can be easily estimated by the Cauchy-Schwarz inequality and Theorem 6.2 to give

$$
\begin{aligned}
\left|M_{1}\right| \leq & \sum_{\mathcal{R} \in \tau_{h}} \int_{\mathcal{R}}\left[\underset{F \in \mathbb{R}^{3 \times 3}}{\operatorname{ess} \sup _{1}}\left\|\nabla_{F} f(x, F)\right\|\right]\left\|\nabla y_{h}(x)-\Pi\left(\nabla y_{h}(x)\right)\right\| d x \\
\leq & \left\{\sum_{\mathcal{R} \in \tau_{h}} \int_{\mathcal{R}}\left[\underset{F \in \mathbb{R}^{3 \times 3}}{\operatorname{ess} \sup _{j}}\left\|\nabla_{F} f(x, F)\right\|\right]^{2} d x\right\}^{\frac{1}{2}} \\
& \cdot\left\{\sum_{\mathcal{R} \in \tau_{h}} \int_{\mathcal{R}}\left\|\nabla y_{h}(x)-\Pi\left(\nabla y_{h}(x)\right)\right\|^{2} d x\right\}^{\frac{1}{2}} \\
\leq & C\|f\|_{\mathcal{V}}\left[\mathcal{E}_{h}\left(y_{h}\right)^{\frac{1}{4}}+\mathcal{E}_{h}\left(y_{h}\right)^{\frac{1}{2}}\right] .
\end{aligned}
$$

To estimate the second term $M_{2}$, we use the identity

$$
\begin{aligned}
f(x, & \Pi(F))-\left[(1-\lambda) f\left(x, F_{0}\right)+\lambda f\left(x, F_{1}\right)\right] \\
& =\frac{1}{|a|^{2}}\left\{a \cdot\left[\Pi(F)-F_{\lambda}\right] n\right\} G_{f}(x), \quad \forall F \in \mathbb{R}^{3 \times 3},
\end{aligned}
$$

to show that

$$
\begin{aligned}
M_{2} \equiv & \sum_{\mathcal{R} \in \tau_{h}} \int_{\mathcal{R}}\left\{f\left(x, \Pi\left(\nabla y_{h}(x)\right)\right)-\left[(1-\lambda) f\left(x, F_{0}\right)+\lambda f\left(x, F_{1}\right)\right]\right\} d x \\
= & \sum_{\mathcal{R} \in \tau_{h}} \int_{\mathcal{R}} \frac{1}{|a|^{2}}\left\{a \cdot\left[\Pi\left(\nabla y_{h}(x)\right)-\nabla y_{h}(x)\right] n\right\} G_{f}(x) d x \\
& \quad+\sum_{\mathcal{R} \in \tau_{h}} \int_{\mathcal{R}} \frac{1}{|a|^{2}}\left\{a \cdot\left[\nabla y_{h}(x)-F_{\lambda}\right] n\right\} G_{f}(x) d x \\
= & \sum_{\mathcal{R} \in \tau_{h}} \int_{\mathcal{R}} \frac{1}{|a|^{2}}\left\{a \cdot\left[\Pi\left(\nabla y_{h}(x)\right)-\nabla y_{h}(x)\right] n\right\} G_{f}(x) d x \\
& \quad+\sum_{\mathcal{R} \in \tau_{h}} \int_{\partial \mathcal{R}} \frac{1}{|a|^{2}}\left\{a \cdot\left[y_{h}(x)-F_{\lambda} x\right]\right\}(n \cdot \nu) G_{f}(x) d S
\end{aligned}
$$




$$
\begin{aligned}
& \quad-\sum_{\mathcal{R} \in \tau_{h}} \int_{\mathcal{R}} \frac{1}{|a|^{2}}\left\{a \cdot\left[y_{h}(x)-F_{\lambda} x\right]\right\}\left[\nabla G_{f}(x) \cdot n\right] d x \\
& \equiv P_{1}+P_{2}+P_{3} .
\end{aligned}
$$

We can estimate $P_{1}$ and $P_{3}$ by the Cauchy-Schwarz inequality, Theorem 6.2 , and Theorem 5.4 by

$$
\begin{aligned}
& \left|P_{1}\right| \leq C\left(\int_{\Omega}\left|G_{f}(x)\right|^{2} d x\right)^{\frac{1}{2}}\left[\mathcal{E}_{h}\left(y_{h}\right)^{\frac{1}{4}}+\mathcal{E}_{h}\left(y_{h}\right)^{\frac{1}{2}}\right], \\
& \left|P_{3}\right| \leq C\left(\int_{\Omega}\left|\nabla G_{f}(x) n\right|^{2} d x\right)^{\frac{1}{2}}\left[\mathcal{E}_{h}\left(y_{h}\right)^{\frac{1}{4}}+\mathcal{E}_{h}\left(y_{h}\right)^{\frac{1}{2}}+h^{\frac{1}{2}}\right] .
\end{aligned}
$$

To estimate $P_{2}$, we denote again $z_{h}(x)=y_{h}(x)-F_{\lambda} x, x \in \Omega$. We rewrite $P_{2}$ as

$$
\begin{aligned}
P_{2} & \equiv \sum_{\mathcal{R} \in \tau_{h}} \sum_{\mathcal{F} \subset \partial \mathcal{R}} \int_{\mathcal{F}} \frac{1}{|a|^{2}}\left[a \cdot z_{h}(x)\right] G_{f}(x)(n \cdot \nu) d S \\
& =\sum_{\mathcal{R} \in \tau_{h}} \sum_{\mathcal{F} \subset \partial \mathcal{R}} \int_{\mathcal{F}} \frac{1}{|a|^{2}}\left\{a \cdot\left[z_{h}(x)-T_{\mathcal{F}}^{a}\left(z_{h}\right)\right]\right\}\left[G_{f}(x)-T_{\mathcal{F}}^{a}\left(G_{f}\right)\right](n \cdot \nu) d S
\end{aligned}
$$

for $y_{h} \in \mathcal{A}_{h}^{a}$ by the definition of $\mathcal{A}_{h}^{a}$ and

$$
\begin{aligned}
P_{2} & \equiv \sum_{\mathcal{R} \in \tau_{h}} \sum_{\mathcal{F} \subset \partial \mathcal{R}} \int_{\mathcal{F}} \frac{1}{|a|^{2}}\left[a \cdot z_{h}(x)\right] G_{f}(x)(n \cdot \nu) d S \\
& =\sum_{\mathcal{R} \in \tau_{h}} \sum_{\mathcal{F} \subset \partial \mathcal{R}} \int_{\mathcal{F}} \frac{1}{|a|^{2}}\left\{a \cdot\left[z_{h}(x)-z_{h}\left(c_{\mathcal{F}}\right)\right]\right\} G_{f}(x)(n \cdot \nu) d S
\end{aligned}
$$

for $y_{h} \in \mathcal{A}_{h}^{p}$ by the definition of $\mathcal{A}_{h}^{p}$. By the same argument as for estimating $J_{2}^{a}$ and $J_{2}^{p}$ in the proof of Theorem 4.2 (cf. (4.10), (4.11), (4.14), and (4.15)) and by Lemma 3.3 and (5.6), we have

$$
\begin{aligned}
\left|P_{2}\right| & \leq C h\left[\sum_{\mathcal{R} \in \tau_{h}} \int_{\mathcal{R}}\left\|\nabla y_{h}(x)-F_{\lambda}\right\|^{2} d x\right]^{\frac{1}{2}}\left[\int_{\Omega}\left|\nabla G_{f}(x)\right|^{2} d x\right]^{\frac{1}{2}} \\
& \leq C h\left[\mathcal{E}_{h}\left(y_{h}\right)^{\frac{1}{2}}+1\right]\left\|\nabla G_{f}\right\|_{0, \Omega} .
\end{aligned}
$$

Finally, the assertion of the theorem follows from (7.5)-(7.10).

\section{ERROR ESTIMATES FOR QUASI-OPTIMAL DEFORMATIONS}

We first establish the existence of finite element energy minimizers as well as the error bound for the corresponding minimum energy.

Theorem 8.1. There exist a constant $C>0$ and $y_{h} \in \mathcal{A}_{h}$ such that

$$
\mathcal{E}_{h}\left(y_{h}\right)=\min _{u_{h} \in \mathcal{A}_{h}} \mathcal{E}_{h}\left(u_{h}\right) \leq C h^{\frac{1}{2}} .
$$

Proof. Fix a mesh $\tau_{h}$. We have by the inverse inequality (3.8), Lemma 5.1, and Theorem 5.4 that

$$
\begin{aligned}
\left\|u_{h}\right\|_{1, \infty, h} & \leq C h^{-\frac{3}{2}}\left\|u_{h}\right\|_{1, h} \\
& \leq C h^{-\frac{3}{2}}\left[\mathcal{E}_{h}\left(u_{h}\right)^{\frac{1}{4}}+\mathcal{E}_{h}\left(u_{h}\right)^{\frac{1}{2}}+1\right], \quad \forall u_{h} \in \mathcal{A}_{h} .
\end{aligned}
$$


Moreover, the continuity of the energy density $\phi$ implies the continuity of the energy functional $\mathcal{E}_{h}$ on the finite-dimensional affine space $\mathcal{A}_{h}$. Therefore, the bound (8.1) implies the existence of a finite element energy minimizer by compactness.

To finish the proof, we need to construct a finite element deformation $y_{h} \in \mathcal{A}_{h}$ such that

$$
\mathcal{E}_{h}\left(y_{h}\right) \leq C h^{\frac{1}{2}} .
$$

This can be demonstrated by an argument similar to that in [8], [32], [31] since the space of our finite element polynomials (3.1) contains all linear polynomials and since the interpolation operator $I_{h}: C(\bar{\Omega}) \rightarrow V_{h}$ satisfies the inequality (3.20).

The number of local minima of the energy functional $\mathcal{E}_{h}$ on $\mathcal{A}_{h}$ grows arbitrarily large as the mesh size $h \rightarrow 0$ [31]. Many of these local minima are approximations on different length scales to the same optimal microstructure [31]. Thus, it is reasonable to give error estimates for finite element approximations $y_{h} \in \mathcal{A}_{h}$ satisfying the quasi-optimality condition

$$
\mathcal{E}_{h}\left(y_{h}\right) \leq \gamma \inf _{u_{h} \in \mathcal{A}_{h}} \mathcal{E}\left(u_{h}\right)
$$

for some constant $\gamma>1$ independent of $h$. Our estimates show that all of the local minima of $\mathcal{E}_{h}$ on $\mathcal{A}_{h}$ which satisfy the quasi-optimality condition give accurate approximations to the energy-minimizing microstructure for the deformation, the volume fractions of the deformation gradients, and the nonlinear integrals of the deformation gradient.

It follows directly from the above theorem and all of the error bounds established in $\S 5, \S 6$, and $\S 7$ that we can obtain the following error estimates for all quasioptimal finite element deformations $y_{h} \in \mathcal{A}_{h}$ and for any family of rectangular meshes $\tau_{h}$ satisfying the quasi-uniformity condition (3.2).

Corollary 8.2. For any $w \in \mathbb{R}^{3}$ satisfying $w \cdot n=0$, there exists a constant $C>0$ such that

$$
\sum_{\mathcal{R} \in \tau_{h}} \int_{\mathcal{R}}\left|\left[\nabla y_{h}(x)-F_{\lambda}\right] w\right|^{2} d x \leq C h^{\frac{1}{4}}
$$

for any $y_{h} \in \mathcal{A}_{h}$ which satisfies the quasi-optimality condition (8.2).

Corollary 8.3. There exists a constant $C>0$ such that

$$
\sum_{\mathcal{R} \in \tau_{h}} \int_{\mathcal{R}}\left|y_{h}(x)-F_{\lambda} x\right|^{2} d x \leq C h^{\frac{1}{4}}
$$

for any $y_{h} \in \mathcal{A}_{h}$ which satisfies the quasi-optimality condition (8.2).

Corollary 8.4. If $\omega \subset \bar{\Omega}$ is a rectangular parallelepiped whose faces are parallel to the coordinate planes, then there exists a constant $C=C(\omega)>0$ such that

$$
\left\|\sum_{\mathcal{R} \in \tau_{h}} \int_{\omega \cap \mathcal{R}}\left[\nabla y_{h}(x)-F_{\lambda}\right] d x\right\| \leq C h^{\frac{1}{16}}
$$

for any $y_{h} \in \mathcal{A}_{h}$ which satisfies the quasi-optimality condition (8.2).

Corollary 8.5. There exists a constant $C>0$ such that

$$
\sum_{\mathcal{R} \in \tau_{h}} \int_{\mathcal{R}}\left\|\nabla y_{h}(x)-\Pi\left(\nabla y_{h}(x)\right)\right\|^{2} d x \leq C h^{\frac{1}{4}}
$$

for any $y_{h} \in \mathcal{A}_{h}$ which satisfies the quasi-optimality condition (8.2). 
Corollary 8.6. If $\omega \subset \bar{\Omega}$ is a rectangular parallelepiped whose faces are parallel to the coordinate planes and $\rho>0$, then there exists a constant $C=C(\omega, \rho)>0$ such that

$$
\left|\frac{\operatorname{meas} \omega_{\rho}^{0}\left(y_{h}\right)}{\operatorname{meas} \omega}-(1-\lambda)\right|+\left|\frac{\operatorname{meas} \omega_{\rho}^{1}\left(y_{h}\right)}{\operatorname{meas} \omega}-\lambda\right| \leq C h^{\frac{1}{16}}
$$

for any $y_{h} \in \mathcal{A}_{h}$ which satisfies the quasi-optimality condition (8.2).

Corollary 8.7. There exists a constant $C>0$ such that

$$
\left|\sum_{\mathcal{R} \in \tau_{h}} \int_{\mathcal{R}}\left\{f\left(x, \nabla y_{h}(x)\right)-\left[(1-\lambda) f\left(x, F_{0}\right)+\lambda f\left(x, F_{1}\right)\right]\right\} d x\right| \leq C\|f\|_{\mathcal{V}} h^{\frac{1}{8}}
$$

for any $f \in \mathcal{V}$ and any $y_{h} \in \mathcal{A}_{h}$ which satisfies the quasi-optimality condition (8.2).

\section{REFERENCES}

[1] Robert Adams, Sobolev spaces, Academic Press, New York, 1975. MR 56:9247

[2] Todd Arbogast and Zhangxin Chen, On the implementation of mixed methods as nonconforming methods for second-order elliptic problems, Math. Comp. 64 (1995), 943-972. MR 95k:65102

[3] John Ball and Richard James, Fine phase mixtures as minimizers of energy, Arch. Rat. Mech. Anal. 100 (1987), 13-52. MR 89c:80005

[4] _ Proposed experimental tests of a theory of fine microstructure and the two-well problem, Phil. Trans. R. Soc. Lond. A 338 (1992), 389-450.

[5] Carsten Carstensen and Petr Plecháč, Numerical solution of the scalar double-well problem allowing microstructure, Math. Comp. 66 (1997), 997-1026. MR 97i:65163

[6] Michel Chipot, Numerical analysis of oscillations in nonconvex problems, Numer. Math. 59 (1991), 747-767. MR 92h:65164

[7] Michel Chipot and Charles Collins, Numerical approximations in variational problems with potential wells, SIAM J. Numer. Anal. 29 (1992), 1002-1019. MR 93j:65171

[8] Michel Chipot, Charles Collins, and David Kinderlehrer, Numerical analysis of oscillations in multiple well problems, Numer. Math. 70 (1995), 259-282. MR 96e:65067

[9] Michel Chipot and David Kinderlehrer, Equilibrium configurations for crystals, Arch. Rat. Mech. Anal. 103 (1988), 237-277. MR 90a:73037

[10] Philippe Ciarlet, The finite element method for elliptic problems, North-Holland, Amsterdam, 1978. MR 58:25001

[11] Charles Collins, Computation of twinning, Microstructure and Phase Transitions (Jerald Ericksen, Richard James, David Kinderlehrer, and Mitchel Luskin, eds.), Springer-Verlag, 1993, IMA Volumes in Mathematics and Its Applications, vol. 54, pp. 39-50. MR 95m:73019

[12] Convergence of a reduced integration method for computing microstructures, SIAM J. Numer. Anal. 35 (1998) (to appear).

[13] Charles Collins, David Kinderlehrer, and Mitchell Luskin, Numerical approximation of the solution of a variational problem with a double well potential, SIAM J. Numer. Anal. 28 (1991), 321-332. MR 92c:73015

[14] Charles Collins and Mitchell Luskin, The computation of the austenitic-martensitic phase transition, Partial Differential Equations and Continuum Models of Phase Transitions (Michel Rascle, Denis Serre, and Marshall Slemrod, eds.), Springer-Verlag, 1989, Lecture Notes in Physics, vol. 344, pp. 34-50. MR 90k:80007

[15] — Numerical modeling of the microstructure of crystals with symmetry-related variants, Proceedings of the US-Japan Workshop on Smart/Intelligent Materials and Systems (Lancaster, Pennsylvania) (I. Ahmad, , M. Aizawa, A. Crowson, and C. Rogers, eds.), Technomic Publishing Co., 1990, pp. 309-318.

[16] _ Optimal order estimates for the finite element approximation of the solution of a nonconvex variational problem, Math. Comp. 57 (1991), 621-637. MR 92a:65276

[17] Charles Collins, Mitchell Luskin, and James Riordan, Computational images of crystalline microstructure, Computing Optimal Geometries (Jean Taylor, ed.), Amer. Math. Soc., 1991, AMS Special Lectures in Mathematics and AMS Videotape Library, pp. 16-18. 
[18] _ Computational results for a two-dimensional model of crystalline microstructure, Microstructure and Phase Transitions (Jerald Ericksen, Richard James, David Kinderlehrer, and Mitchell Luskin, eds.), Springer-Verlag, 1993, IMA Volumes in Mathematics and Its Applications, vol. 54, pp. 51-56. MR 95m:73020

[19] Jerald Ericksen, Constitutive theory for some constrained elastic crystals, Int. J. Solids and Structures 22 (1986), 951-964.

[20] Donald French, On the convergence of finite element approximations of a relaxed variational problem, SIAM J. Numer. Anal. 27 (1990), 419-436. MR 91f:65167

[21] Pierre Gremaud, Numerical analysis of a nonconvex variational problem related to solid-solid phase transitions, SIAM J. Numer. Anal. 31 (1994), 111-127. MR 94m:73023

[22] _ Numerical optimization and quasiconvexity, Euro. J. of Applied Mathematics 6 (1995), 69-82. MR 95m:49003

[23] Richard James and David Kinderlehrer, Theory of diffusionless phase transitions, Partial differential equations and continuum models of phase transitions (M. Rascle, D. Serre, and M. Slemrod, eds.), Springer-Verlag, 1989, Lecture Notes in Physics, vol. 344, pp. 51-84. MR 91e:73011

[24] Petr Klouček, Bo Li, and Mitchell Luskin, Analysis of a class of nonconforming finite elements for crystalline microstructures, Math. Comp. 65 (1996), 1111-1135. MR 97a:73076

[25] Petr Klouček and Mitchell Luskin, The computation of the dynamics of the martensitic transformation, Continuum Mech. Thermodyn. 6 (1994), 209-240. MR 95d:73009

[26] Robert Kohn, The relaxation of a double-well energy, Continuum Mech. Thermodyn. 3 (1991), 193-236. MR 93d:73014

[27] Bo Li, Analysis and computation of martensitic microstructure, Ph.D. thesis, University of Minnesota, 1996.

[28] $\mathrm{Bo} \mathrm{Li}$ and Mitchell Luskin, Finite element approximation of a laminate with varying volume fraction, Tech. Report 1471, IMA, 1997, manuscript.

[29] _ Finite element analysis of microstructure for the cubic to tetragonal transformation, SIAM J. Numer. Anal. 35 (1998) (to appear).

[30] Mitchell Luskin, Numerical analysis of microstructure for crystals with a nonconvex energy density, Progress in Partial Differential Equations: the Metz Surveys (M. Chipot and J. Saint Jean Paulin, eds.), Longman House, 1991, pp. 156-165. MR 94i:73022

[31] _ On the computation of crystalline microstructure, Acta Numerica 5 (1996), 191-258.

[32] _ Approximation of a laminated microstructure for a rotationally invariant, double well energy density, Numer. Math. 75 (1996), 205-221. CMP 97:04

[33] Mitchell Luskin and Ling Ma, Analysis of the finite element approximation of microstructure in micromagnetics, SIAM J. Numer. Anal. 29 (1992), 320-331. MR 92m:65147

[34] Ling Ma and Noel Walkington, On algorithms for non-convex optimization in the calculus of variations, SIAM J. Numer. Anal. 32 (1995), 900-923. MR 96b:49050

[35] Roy Nicolaides and Noel Walkington, Strong convergence of numerical solutions to degenerate variational problems, Math. Comp. 64 (1995), 117-127. MR 95m:65183

[36] P. Pedregal, Numerical approximation of parametrized measures, Num. Funct. Anal. Opt. 16 (1995), 1049-1066. MR 96h:49023

[37] _ On the numerical analysis of non-convex variational problems, Numer. Math. $\mathbf{7 4}$ (1996), 325-336. CMP 97:01

[38] Alfio Quarteroni and Alberto Valli, Numerical approximation of partial differential equations, Springer-Verlag, Berlin, 1994. MR 95i:65006

[39] Rolf Rannacher and Stefan Turek, Simple nonconforming quadrilateral Stokes element, Numer. Meth. for PDEs 8 (1992), 97-111. MR 92i:65170

[40] Tomás Roubíček, A note about relaxation of vectorial variational problems, Calculus of variations, applications and computations (C. Bandle, J. Bemelmans, M. Chipot, J. Saint Jean Paulin, and I. Shafrir, eds.), Longman, 1995, Pitman research notes in mathematical sciences, vol. 326, pp. 208-214. CMP 97:04

[41] _ Numerical approximation of relaxed variational problems, J. Convex Analysis 3 (1996), 329-347. CMP 97:12

[42] W. Rudin, Real and complex analysis, 3rd ed., McGraw-Hill, New York, 1987. MR 88k:00002

[43] Gilbert Strang and George Fix, An analysis of the finite element method, Prentice-Hall, Englewood Cliffs, N.J., 1973. MR 56:1747 
[44] Joseph Wloka, Partial differential equations, Cambridge University Press, Cambridge, 1987. MR 88d:35004

Department of Mathematics, University of California, Los Angeles, 405 Hilgard Avenue, Los Angeles, California 90095-1555

E-mail address: bli@math.ucla.edu

School of Mathematics, University of Minnesota, 206 Church Street, S.E., MinneapoLIS, Minnesota 55455

E-mail address: luskin@math.umn.edu 